JOURNAL OF THE

AMERICAN MATHEMATICAL SOCIETY

Volume 13, Number 3, Pages 553-594

S 0894-0347(00)00332-5

Article electronically published on April 26, 2000

\title{
GENERALIZED GROUP CHARACTERS AND COMPLEX ORIENTED COHOMOLOGY THEORIES
}

MICHAEL J. HOPKINS, NICHOLAS J. KUHN, AND DOUGLAS C. RAVENEL

\section{Contents}

1. Introduction

2. Complex oriented descent and equivariant bundles

3. Rational equivariant stable homotopy and Artin's Theorem 564

4. Complex oriented Euler characteristics 568

5. Formal groups and $E^{*}(B A) \quad 573$

6. Generalized characters $\quad 578$

7. Good groups $\quad 588$

Acknowledgements $\quad 592$

References $\quad 592$

\section{INTRODUCTION}

Let $B G$ be the classifying space of a finite group $G$. Given a multiplicative cohomology theory $E^{*}$, the assignment

$$
G \longmapsto E^{*}(B G)
$$

is a functor from groups to rings, endowed with induction (transfer) maps. In this paper we investigate these functors for complex oriented cohomology theories $E^{*}$, particularly $p$-complete theories with an associated formal group of height $n$.

We briefly remind our readers of the terms in this last sentence. A multiplicative cohomology theory $E^{*}$ is complex oriented if there exists a class $x \in E^{2}\left(\mathbb{C} P^{\infty}\right)$ that restricts to a generator of the free rank one $E^{*}=E^{*}(\mathrm{pt})-$ module $\tilde{E}^{2}\left(\mathbb{C} P^{1}\right)$. Such a class $x$ is called a complex orientation of $E$. An orientation allows for the construction in $E^{*}$-theory of Chern classes for complex vector bundles. Furthermore, the behavior of these Chern classes under the tensor product of bundles is determined by an associated formal group law over the ring $E^{*}$. When localized at a prime $p$, such formal group laws are classified by 'height'. Under the completeness hypotheses we will be assuming, a height $n$ formal group law will force an element $v_{n} \in E^{2-2 p^{n}}$ to be invertible, and thus we may informally refer to such theories as $v_{n}$-periodic.

Received by the editors July 20, 1999 and, in revised form, January 28, 2000.

2000 Mathematics Subject Classification. Primary 55N22; Secondary 55N34, 55N91, 55R35, $57 \mathrm{R} 85$.

All three authors were partially supported by the National Science Foundation.

(C)2000 American Mathematical Society 
Our work was inspired by complex K-theory, which, when localized at a prime $p$, is $v_{1}$-periodic. In this case, Atiyah Atiyah showed that $K^{*}(B G)$ is isomorphic to the completion of the complex representation ring $R(G)$, in the topology induced by the ideal of virtual representations of degree 0 . The ring $R(G)$ can, of course, be studied via group characters, leading to many well-known results: the cyclic subgroups form a detecting family, the rank of $R(G)$ is the number of conjugacy classes, etc.

It often turns out that the ring $E^{*}(B G)$ can also be studied with characters. For suitable $v_{n}$-periodic theories $E^{*}$, these characters assign to each conjugacy class of commuting $n$-tuples of $p$-elements of $G$ an element of a ring we associate to $E^{*}$. Furthermore, one can extend these constructions to detect elements in $E^{*}\left(E G \times_{G} X\right)$ for finite $G-\mathrm{CW}$ complexes $X$, where now the 'character ring' also depends on the abelian fixed point data of $X$. As a result, almost anything that can be said about representation rings and equivariant $\mathrm{K}$-theory has an analogue for $E^{*}(B G)$ and $E^{*}\left(E G \times_{G} X\right)$.

Versions of our basic theorems date from 1986 and 1987, and many readers will be aware of earlier drafts of this paper dating from 1989 and 1992. We thank such readers for their patience, and hope they will appreciate our more accurate arguments, improved organization, and slightly strengthened theorems.

A standing convention in this paper is that we are working in categories of graded objects. Thus rings are graded, tensor products of graded objects are graded in the standard way, ideals in a ring are assumed to be generated by homogeneous elements, homomorphisms preserve grading, etc.

1.1. A generalized Artin's theorem. Our first theorem is valid for all complex oriented theories, and highlights the privileged role played by the abelian groups.

Let $\mathcal{A}(G)$ be the category having objects the abelian subgroups of $G$, and with morphisms from $B$ to $A$ being the $G$-maps from $G / B$ to $G / A$. (Thus this is a full subcategory of the standard orbit category.) Given a $G$-space $X$, a morphism $G / B \rightarrow G / A$ in $\mathcal{A}(G)$ induces maps $G \times_{B} X \rightarrow G \times_{A} X$ and $X^{A} \rightarrow X^{B}$ in the usual way.

If $E^{*}$ is a generalized cohomology theory, the $G$-maps $G \times{ }_{A} X \rightarrow X$ induce a

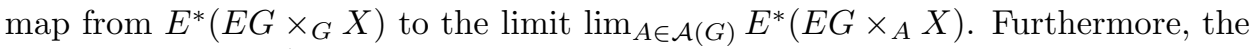
$G$-maps $G / A \times X^{A} \rightarrow G \times{ }_{A} X$ induce a map from $\lim _{A \in \mathcal{A}(G)} E^{*}\left(E G \times_{A} X\right)$ to the end $\int_{A \in \mathcal{A}(G)} E^{*}\left(B A \times X^{A}\right) !$

Theorem A. Let $E$ be a complex oriented cohomology theory. For any finite group $G$ and finite $G-C W$ complex $X$, each of the natural maps

$$
E^{*}\left(E G \times{ }_{G} X\right) \rightarrow \lim _{A \in \mathcal{A}(G)} E^{*}\left(E G \times{ }_{A} X\right) \rightarrow \int_{A \in \mathcal{A}(G)} E^{*}\left(B A \times X^{A}\right)
$$

becomes an isomorphism after inverting the order of $G$. In particular,

$$
\frac{1}{|G|} E^{*}(B G) \rightarrow \lim _{A \in \mathcal{A}(G)} \frac{1}{|G|} E^{*}(B A)
$$

is an isomorphism.

\footnotetext{
${ }^{1} \mathrm{~A} G$-map $\alpha: G / A_{1} \longrightarrow G / A_{2}$ induces $\alpha_{*}: E^{*}\left(B A_{1} \times X^{A_{1}}\right) \rightarrow E^{*}\left(B A_{1} \times X^{A_{2}}\right)$ and $\alpha^{*}: E^{*}\left(B A_{2} \times X^{A_{2}}\right) \longrightarrow E^{*}\left(B A_{1} \times X^{A_{2}}\right)$. The end $\int_{A \in \mathcal{A}(G)} E^{*}\left(B A \times X^{A}\right)$ is then defined to be the subring of $\prod_{A \in \mathcal{A}(G)} E^{*}\left(B A \times X^{A}\right)$ consisting of those elements $\left(x_{A}\right)$ in this product such that $\alpha_{*}\left(x_{A_{1}}\right)=\alpha^{*}\left(x_{A_{2}}\right)$ for all $\alpha: G / A_{1} \longrightarrow G / A_{2}$.
} 
This is an analogue of Artin's theorem [Serre, Chapter 9]: For any finite group $G$, the natural map

$$
\frac{1}{|G|} R(G) \longrightarrow \lim _{C \in \mathcal{C}(G)} \frac{1}{|G|} R(C)
$$

is an isomorphism, where $\mathcal{C}(G)$ is the full subcategory of $\mathcal{A}(G)$ having the cyclic subgroups as objects.

In the case of ordinary cohomology, Theorem A offers no information since $\tilde{H}^{*}(B G)$ is all $|G|$-torsion. However $K^{*}(B G)$ is known to be torsion free, and $M U^{*}(B G)$ is presumed to often be.

In proving this theorem, we use the most fundamental idea from the theory of complex oriented cohomology: the notion of complex oriented descent (a.k.a. the splitting principle). The other ingredient is a modest amount of equivariant stable homotopy, together with related ideas from the theory of Mackey functors.

Section 2 contains what we need about complex oriented descent. Theorem A is proved in section 3 as a special case of a more general theorem, Theorem 3.3 This theorem has other interesting corollaries. For example, it implies that the map between equivariant bordism rings

$$
M U_{G}^{*} \rightarrow \lim _{A \in \mathcal{A}(G)} M U_{A}^{*}
$$

also becomes an isomorphism after inverting the order of $G$.

1.2. Morava $\mathbf{K}$-theory Euler characteristics. Now we describe the computation that led to this project. Fixing a prime $p$, let $K(n)^{*}$ denote the $n^{\text {th }}$ Morava Ktheory at $p[\operatorname{Rav} 86]$. This is a complex oriented theory with coefficients $K(n)^{*}$ equal to the graded field $\mathbb{F}_{p}\left[v_{n}, v_{n}^{-1}\right]$. The third author noted in $[\operatorname{Rav} 82]$ that $K(n)^{*}(B G)$ is a finite dimensional vector space over $K(n)^{*}$, and asked for its dimension. The second author Kuhn1 computed this in the special case when $G$ has abelian $p$ Sylow subgroups, with the answer involving certain orbits of $n$-tuples of elements in a fixed Sylow subgroup 2

Our partial answer to the general question is the following. Let $G_{n, p}$ denote the set of $n$-tuples of commuting elements each of which has order a power of $p$. The group $G$ acts on $G_{n, p}$ by conjugation: if $\alpha=\left(g_{1}, \ldots, g_{n}\right)$, define $g \cdot \alpha=$ $\left(g g_{1} g^{-1}, \ldots, g g_{n} g^{-1}\right)$.

Theorem B (Part 1). The Morava K-theory Euler characteristic

$$
\chi_{n, p}^{G}=\operatorname{dim} K(n)^{\text {even }}(B G)-\operatorname{dim} K(n)^{\text {odd }}(B G)
$$

is equal to the number of $G$-orbits in $G_{n, p}$.

The proof of Theorem $\mathrm{B}$ involves counting these orbits by means of Möbius functions on the partially ordered set of abelian subgroups of $G$. We also generalize our computation to a computation of

$$
\chi_{n, p}^{G}(X)=\operatorname{dim} K(n)^{\text {even }}\left(E G \times_{G} X\right)-\operatorname{dim} K(n)^{\text {odd }}\left(E G \times_{G} X\right),
$$

for any finite $G-\mathrm{CW}$ complex $X$.

\footnotetext{
${ }^{2}$ Let $W_{G}(A)=N_{G}(A) / A$, where the abelian group $A$ is a $p$-Sylow subgroup. Then the dimension of $K(n)^{*}(B G)$ is the number of $W_{G}(A)$-orbits in $A^{n}$. It is an exercise with the Sylow theorems to check that this agrees with Theorem B.
} 
Our Möbius functions are defined using the usual Euler characteristic as follows. For all abelian subgroups $A \leq G$ and finite $G-\mathrm{CW}$ complexes $X$, an integer $\mu_{A}^{\mathbb{C}}(X)$ is defined by downward induction on $A$ by the equation

$$
\sum_{\substack{A \leq B \\ B \text { abelian }}} \mu_{B}^{\mathbb{C}}(X)=\chi\left(X^{A}\right) .
$$

Our formula for $\chi_{n, p}^{G}(X)$ is then

Theorem B (Part 2).

$$
\chi_{n, p}^{G}(X)=\sum_{A \leq G} \frac{|A|}{|G|}\left|A_{(p)}\right|^{n} \mu_{A}^{\mathbb{C}}(X) .
$$

Here the sum is over the abelian subgroups of $G$, and $A_{(p)}$ denotes the $p$-Sylow subgroup of $A$.

The function $\chi_{n, p}^{G}$ on finite $G-\mathrm{CW}$ complexes is an example of an 'additive invariant' in the sense of tom Dieck [TtD:87, p.227]. Our function satisfies an extra condition due to complex oriented descent. In $\$ 4$ we develop the general theory of such complex oriented additive functions. Theorem B then follows from this, together with one bit of special information about $K^{*}(n)$ : if $A$ is an abelian group, $K(n)^{*}(B A)$ is an $\left|A_{(p)}\right|^{n}$ dimensional $K(n)^{*}$-vector space concentrated in even degrees.

1.3. Generalized characters. Theorem $\AA$ can be interpreted in terms of characters - at the cost of adhering more structure to $E^{*}$.

To motivate our result, recall that classical characters for finite groups are defined in the following situation. Let $L$ be the smallest characteristic 0 field containing all roots of unity, and, if $G$ is a finite group, let $C l(G ; L)$ be the ring of class functions on $G$ with values in $L$. The units in the profinite integers $\hat{\mathbb{Z}}$ act on $L$ as the Galois group over $\mathbb{Q}$. Observing that $G=\operatorname{Hom}(\hat{\mathbb{Z}}, G)$, the set of continuous homomorphisms, one sees that $\hat{\mathbb{Z}}^{\times}$also acts naturally on $G$, and thus on $C l(G ; L)$ : given $\phi \in \hat{\mathbb{Z}}, g \in G$, and $\chi \in C l(G ; L)$, one lets $(\phi \cdot \chi)(g)=\phi\left(\chi\left(\phi^{-1}(g)\right)\right)$. The character map is a ring homomorphism 3

$$
\chi: R(G) \rightarrow C l(G ; L)^{\hat{\mathbb{Z}}},
$$

and this induces isomorphisms

$$
\chi: L \otimes R(G) \simeq C l(G ; L)
$$

and

$$
\chi: \mathbb{Q} \otimes R(G) \simeq C l(G ; L)^{\hat{\mathbb{Z}}} .
$$

(See [Serre, Theorem 25] for this last statement.)

Let $E^{*}$ be a complex oriented theory, with associated formal group law $F$ associated to a fixed orientation $x \in E^{2}\left(\mathbb{C} P^{\infty}\right)$. Suppose the graded ring $E^{*}$ and $F$ satisfy

- $E^{*}$ is local with maximal ideal $\mathfrak{m}$, and complete in the $\mathfrak{m}$-adic topology.

- The graded residue field $E^{*} / \mathfrak{m}$ has characteristic $p>0$.

\footnotetext{
${ }^{3}$ We apologize for our excessive use of the symbol $\chi$. We use it to denote both group characters and Euler characteristics, as dictated by traditions in representation theory and topology. In context, we hope there is no confusion.
} 
- $p^{-1} E^{*}$ is not zero.

- The mod $\mathfrak{m}$ reduction of $F$ has height $n<\infty$ over $E^{*} / \mathfrak{m}$. (See \$5.1)

We define $L\left(E^{*}\right)$, the analogue of $L$, in the following way. The inverse system

$$
\cdots \rightarrow\left(\mathbb{Z} / p^{r+1}\right)^{n} \rightarrow\left(\mathbb{Z} / p^{r}\right)^{n} \rightarrow \cdots
$$

induces a direct system of $E^{*}$-algebras

$$
\cdots \rightarrow E^{*}\left(B\left(\mathbb{Z} / p^{r}\right)^{n}\right) \rightarrow E^{*}\left(B\left(\mathbb{Z} / p^{r+1}\right)^{n}\right) \rightarrow \ldots
$$

and we let $E_{\text {cont }}^{*}\left(B \mathbb{Z}_{p}^{n}\right)$ denote the colimit. A continuous homomorphism from the $n$-fold product of the $p$-adic integers to the circle $\alpha: \mathbb{Z}_{p}^{n} \rightarrow S^{1}$ will induce a map $\alpha^{*}: E^{*}\left(\mathbb{C} P^{\infty}\right) \rightarrow E_{\text {cont }}^{*}\left(B \mathbb{Z}_{p}^{n}\right)$ and we let $c_{1}(\alpha)=\alpha^{*}(x) \in E_{\text {cont }}^{2}\left(B \mathbb{Z}_{p}^{n}\right)$. Now let $L\left(E^{*}\right)=S^{-1} E_{\text {cont }}^{*}\left(B \mathbb{Z}_{p}^{n}\right)$ where $S$ is the set of $c_{1}(\alpha)$ corresponding to nonzero homomorphisms $\alpha$. Note that the continuous automorphism group $\operatorname{Aut}\left(\mathbb{Z}_{p}^{n}\right)$ acts on $L\left(E^{*}\right)$ as a ring of $E^{*}$-algebra maps.

The analogue of $C l(G ; L)$ will be $C l_{n, p}\left(G ; L\left(E^{*}\right)\right)$, defined to be the ring of functions $\chi: G_{n, p} \rightarrow L\left(E^{*}\right)$ stable under $G$-orbits. Noting that

$$
G_{n, p}=\operatorname{Hom}\left(\mathbb{Z}_{p}^{n}, G\right),
$$

one sees that $\operatorname{Aut}\left(\mathbb{Z}_{p}^{n}\right)$ acts on $G_{n, p}$, and thus on $C l_{n, p}\left(G ; L\left(E^{*}\right)\right)$ as a ring of $E^{*}-$ algebra maps: given $\phi \in \operatorname{Aut}\left(\mathbb{Z}_{p}^{n}\right), \alpha \in G_{n, p}$, and $\chi \in C l_{n, p}\left(G ; L\left(E^{*}\right)\right)$, one lets $(\phi \cdot \chi)(\alpha)=\phi\left(\chi\left(\phi^{-1}(\alpha)\right)\right)$.

More generally, if $X$ is a finite $G-\mathrm{CW}$ complex, let

$$
\operatorname{Fix}_{n, p}(G, X)=\coprod_{\alpha \in \operatorname{Hom}\left(\mathbb{Z}_{p}^{n}, G\right)} X^{\operatorname{Im}(\alpha)} .
$$

This is a space with commuting actions of $G$ and $\operatorname{Aut}\left(\mathbb{Z}_{p}^{n}\right)$. Let

$$
C l_{n, p}\left(G, X ; L\left(E^{*}\right)\right)=L\left(E^{*}\right) \otimes_{E^{*}} E^{*}\left(\operatorname{Fix}_{n, p}(G, X)\right)^{G} .
$$

This is again an $E^{*}$-algebra acted on by $\operatorname{Aut}\left(\mathbb{Z}_{p}^{n}\right)$.

We define our character map

$$
\chi_{n, p}^{G}: E^{*}\left(E G \times_{G} X\right) \rightarrow C l_{n, p}\left(G, X ; L\left(E^{*}\right)\right)^{\operatorname{Aut}\left(\mathbb{Z}_{p}^{n}\right)}
$$

as follows. Using a Künneth isomorphism

$$
E^{*}\left(B\left(\mathbb{Z} / p^{m}\right)^{n} \times X^{\operatorname{Im}(\alpha)}\right) \simeq E^{*}\left(B\left(\mathbb{Z} / p^{m}\right)^{n}\right) \otimes_{E^{*}} E^{*}\left(X^{\operatorname{Im}(\alpha)}\right)
$$

available in our situation (see Corollary 5.11), a homomorphism $\alpha \in \operatorname{Hom}\left(\mathbb{Z}_{p}^{n}, G\right)$ induces

$$
E^{*}\left(E G \times_{G} X\right) \rightarrow E_{\text {cont }}^{*}\left(B \mathbb{Z}_{p}^{n}\right) \otimes_{E^{*}} E^{*}\left(X^{\operatorname{Im}(\alpha)}\right) \rightarrow L\left(E^{*}\right) \otimes_{E^{*}} E^{*}\left(X^{\operatorname{Im}(\alpha)}\right) .
$$

This will be $\chi_{n, p}^{G}(\alpha)$, the component of $\chi_{n, p}^{G}$ indexed by $\alpha$.

Our main theorem is then

Theorem C. The invariant ring $L\left(E^{*}\right)^{\operatorname{Aut}\left(\mathbb{Z}_{p}^{n}\right)}=p^{-1} E^{*}$, and $L\left(E^{*}\right)$ is faithfully flat over $p^{-1} E^{*}$. The character map $\chi_{n, p}^{G}$ induces isomorphisms

$$
\chi_{n, p}^{G}: L\left(E^{*}\right) \otimes_{E^{*}} E^{*}\left(E G \times_{G} X\right) \simeq C l_{n, p}\left(G, X ; L\left(E^{*}\right)\right)
$$

and

$$
\chi_{n, p}^{G}: p^{-1} E^{*}\left(E G \times_{G} X\right) \simeq C l_{n, p}(G, X ; L(E))^{\operatorname{Aut}\left(\mathbb{Z}_{p}^{n}\right)} .
$$


In particular, there are isomorphisms

$$
\chi_{n, p}^{G}: L\left(E^{*}\right) \otimes_{E^{*}} E^{*}(B G) \simeq C l_{n, p}\left(G ; L\left(E^{*}\right)\right)
$$

and

$$
\chi_{n, p}^{G}: p^{-1} E^{*}(B G) \simeq C l_{n, p}\left(G ; L\left(E^{*}\right)\right)^{\operatorname{Aut}\left(\mathbb{Z}_{p}^{n}\right)} .
$$

Theorem $[$ applies to many cohomology theories:

- The 'completions of complex cobordism' introduced by Morava in Mor78.

- The ' $I_{n}$-adically completed' version of $E(n)$ studied by A. Baker and Würgler [BW89. They consider $\hat{E}(n)$, the Bousfield localization of $E(n)$ with respect to $K(n)$, where $E^{*}(n)=\mathbb{Z}_{(p)}\left[v_{1}, \ldots, v_{n}, v_{n}^{-1}\right]$, as a module over $B P^{*}=$ $\mathbb{Z}_{(p)}\left[v_{1}, v_{2}, \ldots\right]$. They show that $\hat{E}(n)^{*}$ is the completion of $E^{*}(n)$ with respect to the ideal $I_{n}=\left(p, v_{1}, \ldots, v_{n-1}\right)$.

- The 'integral lifts' of the Morava K-theories studied by Morava in [Mor88. The coefficients are $W\left(\mathbb{F}_{p^{n}}\right)\left[u, u^{-1}\right]$ where $W\left(\mathbb{F}_{p^{n}}\right)$ denotes the Witt vectors for the finite field, and $u$ has degree -2 .

- The completion of elliptic cohomology [LRS at any maximal ideal (see Bak98] and [Hop89]).

- The theories $E_{n}$ studied by Hopkins and Miller with coefficients

$$
\left.W\left(\mathbb{F}_{p^{n}}\right)\left[w_{1}, \ldots, w_{n-1}\right]\right]\left[u, u^{-1}\right]
$$

$\left(w_{i}\right.$ has degree 0$)$. These spectra have been shown to admit $E_{\infty}$-ring structures, thus one has a good theory of power operations which our characters can be used to explore.

Section[5]contains the prerequisites we need about formal group laws and $E^{*}(B A)$ for $A$ abelian. Properties of $L\left(E^{*}\right)$ are then developed in section 6 , along with a proof of Theorem C.

1.4. A formula for induction. It is useful to have a formula for the character of an 'induced' cohomology class. Recall that, for $H \leq G, X$ a $G$-space, and any cohomology theory $E^{*}$, there is a transfer map [Ada78. Chapter 4]

$$
\operatorname{Tr}: E^{*}\left(E H \times_{H} X\right) \longrightarrow E^{*}\left(E G \times_{G} X\right) .
$$

Theorem D. Let $x$ be an element of $E^{*}\left(E H \times_{H} X\right)$. Then

$$
\chi_{n, p}^{G}(\alpha)\left(\operatorname{Tr}^{*}(x)\right)=\sum_{g H \in(G / H)^{I m(\alpha)}} \chi_{n, p}^{H}(g \cdot \alpha)(x) .
$$

In this formula, $\alpha: \mathbb{Z}_{p}^{n} \rightarrow G$ is a homomorphism, and $g \cdot \alpha$ means to follow this with conjugation by $g$.

This formula generalizes the classic situation [Serre, p.30], and is proved at the end of section 6 .

As a simple application, Theorem D can be used to compute the kernel of the stable Hurewicz map

$$
\pi_{S}^{0}(B G) \rightarrow M U^{0}(B G)
$$

up to finite index. This example appears as Example 6.16] and is related to work of Stretch [Stre81] and Laitinen Lai79] on the Segal Conjecture. 
1.5. When is $K(n)^{*}(B G)$ concentrated in even degrees? In contemplating Theorems $\mathrm{A}$ and $\mathrm{C}$, it is natural to wonder if there is any $|G|$-torsion in $E^{*}(B G)$ for the theories $E^{*}$ of interest. Suppose, in particular, $E^{*}$ is a $p$-complete integral lift of $K(n)^{*}$, that is, $E^{*}(X ; \mathbb{Z} / p)=K(n)^{*}(X) .4$ Then an argument with Bocksteins shows that $E^{*}(B G)$ will be $p$-torsion free if and only if $K(n)^{*}(B G)$ is concentrated in even degrees. Similarly, a more elaborate argument with Bockstein-like spectral sequences shows that if $K(n)^{*}(B G)$ is concentrated in even degrees, then $E_{n}^{*}(B G)$ is torsion free, where $E_{n}$ is the important theory mentioned above. (See e.g. Stri98] for this type of argument.)

In the classic case of $K$-theory, along with proving that $K^{0}(B G)$ is the completion of the representation ring, Atiyah showed that $K^{1}(B G)=0$. Many readers of this paper will know that, inspired by this, the authors originally conjectured that $K(n)^{\text {odd }}(B G)=0$ for all $n$ and $G$. This conjecture was disproved by I. Kriz Kri97] in the case $p=3, n=2$, and with $G$ the 3 -Sylow subgroup of $G L_{4}(\mathbb{Z} / 3)$, a group of order $3^{6}$. (Kriz and K. Lee now have examples for all odd $p$ [KL98.)

From the beginnings of this project, the authors knew that a critical part of Atiyah's inductive argument failed to generalize to the $n \geq 2$ case: if $G$ is a $p$ group, $K^{0}(B G)$ is a permutation $\operatorname{Aut}(G)$-module. This is also the point that Kriz exploits as his example arises from an extension

$$
1 \rightarrow H \rightarrow G \rightarrow \mathbb{Z} / p \rightarrow 1
$$

in which $E^{*}(B H)$ is not a permutation $\mathbb{Z} / p$-module, where $E^{*}$ is (roughly) an integral lift of $K(2)$.

This permutation module problem suggested that perhaps $K(n)^{*}(B G)$ could be proven to be concentrated in even degrees if $G$ is built up out of extensions related to permutations. We have a result along these lines.

We define a finite group $G$ to be good if $K(n)^{*}(B G)$ is generated as a $K(n)^{*}$ module by transfers of Euler classes of complex representations of subgroups. In particular, if $G$ is good, then $K(n)^{*}(B G)$ is concentrated in even degrees.

Theorem E. i) Every finite abelian group is good.

ii) If $G_{1}$ and $G_{2}$ are good, then so is their product $G_{1} \times G_{2}$.

iii) $G$ is good if its $p$-Sylow subgroup is good.

iv) If $G$ is good, then so is $\mathbb{Z} / p \nmid G$, the wreath product arising as the extension

$$
1 \rightarrow G^{p} \rightarrow \mathbb{Z} / p \curlywedge G \rightarrow \mathbb{Z} / p \rightarrow 1 .
$$

This will be proved in section 7. The first of these statements is well known, and the second and third are easy to verify. It is the last statement that makes our notion interesting. In particular, since the $p$-Sylow subgroup of any symmetric group is a product of iterated wreath products of $\mathbb{Z} / p$, we have proved that symmetric groups $\Sigma_{k}$ are all good.

In Proposition [7.10, we will show that calculations by Tezuka-Yagita [TY] imply that all groups of order $p^{3}$ are good. In recent years, the list of known good groups has been expanded by various people; see e.g. [Kri97, Tan95.

J. Hunton [Hun90] independently has shown that the symmetric groups have no odd dimensional Morava $\mathrm{K}$-theory by defining a variant of our notion good and then proving an analogue of the last statement of our theorem.

\footnotetext{
${ }^{4}$ It would also suffice to have the spectrum $E / p$ be a $K(n)$-module, necessarily free, on even dimensional classes.
} 
1.6. Historical remarks. Since this paper has taken so long to be put in final form, it is perhaps appropriate to comment on its history.

The idea that the correct domain for our characters should be $G_{n, p}$ was inspired by the 1985 work [Kuhn1]. At the January 1986 A.M.S. meeting in New Orleans, the third author conjectured that the rank of $K(n)^{*}(B G)$ was equal to the number of $G$-orbits in $G_{n, p}$. Soon after, the first and third authors realized that LubinTate theory together with a Vandermonde determinant argument (appearing here in the proof of Proposition 6.2 led to some sort of version of Theorem C We soon derived the formula for the transfer, and a first proof of Theorem B was discovered by the first author. Our work, as of mid 1986, was publicly presented in an informal evening talk by Hopkins on July 31, 1986 at the algebraic topology conference in Arcata, CA.

It is fair to say that, at that time, the necessary hypotheses regarding completion had not been accurately sorted out, nor had adequate attention been payed to the flatness of the extension of scalars to $L\left(E^{*}\right)$.

In 1986, it seemed a big presumption to assume the coefficient ring $E^{*}$ was complete. Today this seems much less a problem, as numerous interesting results exploiting this hypothesis have been proven, and interesting examples developed, starting with [BW89, Mor88]. Thus, during the first year of the project, $E^{*}$ was just assumed to be local, but not necessarily complete, and we attempted to prove theorems about $E^{*}(B G)^{\wedge}$, where ${ }^{\wedge}$ is algebraic completion at the maximal ideal. This seems to lead into a thicket of questions about the exactness of completion in non-Noetherian settings, and during 1986-87, we became convinced that the hypothesis of completeness was hard to avoid.

The formalities of our character rings from the Mackey functor point of view were investigated by the second author during a 1986-87 visit to Cambridge University. Theorem $\mathrm{A}$ was discovered as a consequence, and the first formal presentation of this theorem was at the Oxford Topology Seminar of June 8, 1987.5 Related observations made at this time are more fully discussed in [Kuh89].6

Armed with Theorem $\mathrm{A}$, it was initially unclear if one really needed to prove the flatness of $L\left(E^{*}\right)$ as an $E^{*}$-module - and one doesn't, if one is content with describing $E^{*}(B G)$ and not $E^{*}\left(E G \times_{G} X\right)$. (See Remark 6.11) However the demands of subsequent work by Hopkins and his younger colleagues at M.I.T. made it clear that a good understanding of this ring was important. By 1992, the algebro-geometric point of view had become more conceptually important, and has been crucial in subsequent work by Ando, Hopkins, Strickland, and others.

${ }^{5}$ This was a talk by Kuhn joint with J.F. Adams (!).

${ }^{6}$ This includes an analogue of Theorem [C for equivariant K-theory: there is a character map

$$
\chi: \mathbb{C} \otimes K_{G}(X) \longrightarrow\left(\prod_{g \in G} \mathbb{C} \otimes K\left(X^{g}\right)\right)^{G}
$$

that is an isomorphism for all finite $G-\mathrm{CW}$ complexes $X$. Though this formula also appeared in a letter from Kuhn to G. Segal dated December 4, 1986, it was widely advertised by Hirzebruch as a 'recent formula of Atiyah and Segal' after the publication of [AS89]. But precedence for such formulae seems to be due to tom Dieck: in his 1979 book, he gave the closely related formula [Die79, p.198]

$$
\frac{1}{|G|} K_{G}(X) \simeq\left(\prod_{C} S_{C}^{-1} R(C) \otimes K\left(X^{C}\right)\right)^{G},
$$

where the product runs over the cyclic subgroups of $G$, and $S_{C}$ is the set of Euler classes of nontrivial ireducible $C$-modules. 
Our natural description of the spectrum of $E^{*}(B A)$ (Proposition 5.12) reflects this changing point of view.

In recent years, the proofs of Theorems A and B have been significantly altered, leading to the current nearly axiomatic presentations. The refinement of Theorem C using $\operatorname{Aut}\left(\mathbb{Z}_{p}^{n}\right)$ dates from the early 1990's.

The formulation and proof of Theorem E is due to the third author around 1988.

Expository articles about aspects of this work have been written by all of us: Hop89, Kuh89, HKR92.

From the beginning of this project, it has been noticed that, if $Y=E G \times{ }_{G} X, E^{*}$ is ' $v_{n}$-periodic' and $D^{*}$ is ' $v_{n-1}$-periodic', then there seems to be a close relationship between

$$
E^{*}(Y) \text { and } D^{*}\left(Y^{S^{1}}\right)
$$

Similarly, if $G$ is a $p$-group and $T^{n}$ is the $n$-torus, there is a natural isomorphism of $L^{*}\left(E^{*}\right)$ algebras

$$
L\left(E^{*}\right) \otimes_{E^{*}} E^{*}(B G) \simeq H^{*}\left(B G^{T^{n}} ; L\left(E^{*}\right)\right) .
$$

When $n=2$, this fits well with work of Taubes Tau and Bott-Taubes [BT] on the elliptic genus. However, it has yet to be explained in a satisfactory manner, and, to get to the heart of the matter, there is not yet a good explanation of what basic geometric structures, analogous to vector bundles, our characters are giving us information about. Perhaps in the next millennium we will learn more.

\section{Complex ORIENTED DESCENT AND EQUivariant BUNDles}

2.1. A useful way to construct equalizers. At various times in this paper, we describe objects as equalizers. Without exception, they all arise using a general categorical procedure we describe in this subsection.

The situation is the following. One has

- Two categories $\mathcal{C}$ and $\mathcal{A}$.

- Two functors $\mathcal{F}: \mathcal{C} \rightarrow \mathcal{C}$ and $\mathcal{H}: \mathcal{C}^{o p} \rightarrow \mathcal{A}$.

- Two natural transformations $p(X): \mathcal{F}(X) \rightarrow X$ and $r(X): \mathcal{H}(\mathcal{F}(X)) \rightarrow$ $\mathcal{H}(X)$.

These are required to satisfy the following two properties:

$$
\begin{aligned}
& \text { For all } X \in \mathcal{C}, p(X) \circ p(\mathcal{F}(X))=p(X) \circ \mathcal{F}(p(X)): \mathcal{F}(\mathcal{F}(X)) \rightarrow X \\
& \text { For all } X \in \mathcal{C}, r(X) \circ \mathcal{H}(p(X))=1: \mathcal{H}(X) \rightarrow \mathcal{H}(X) .
\end{aligned}
$$

Proposition 2.3. In this situation, there is an equalizer diagram

$$
\mathcal{H}(X) \rightarrow \mathcal{H}(\mathcal{F}(X)) \rightrightarrows \mathcal{H}(\mathcal{F}(\mathcal{F}(X))) .
$$

Proof. Applying $\mathcal{H}$ to (2.1) shows that the two composites are equal. To finish the proof, we show that if $\alpha: A \rightarrow \mathcal{H}(\mathcal{F}(X))$ is any morphism in $\mathcal{A}$ satisfying

$$
\mathcal{H}(p(\mathcal{F}(X))) \circ \alpha=\mathcal{H}(\mathcal{F}(p(X))) \circ \alpha: \mathcal{H}(\mathcal{F}(X)) \rightarrow \mathcal{H}(\mathcal{F}(\mathcal{F}(X))),
$$

then $\alpha$ factors uniquely through $\mathcal{H}(p(X))$; more precisely,

$$
\alpha=\mathcal{H}(p(X)) \circ \beta, \text { where } \beta=r(X) \circ \alpha: A \rightarrow \mathcal{H}(X) .
$$


To check this, we compute:

$$
\begin{aligned}
\mathcal{H}(p(X)) \circ r(X) \circ \alpha & =r(\mathcal{F}(X)) \circ \mathcal{H}(\mathcal{F}(p(X))) \circ \alpha \\
& =r(\mathcal{F}(X)) \circ \mathcal{H}(p(\mathcal{F}(X))) \circ \alpha \\
& =\alpha .
\end{aligned}
$$

Here the first equality is a consequence of the naturality of $r$, the second is true by the hypothesis on $\alpha$, and the third follows from (2.2).

2.2. Cohomology of flag bundles. Let $\xi$ be an $m$-dimensional complex vector bundle over a space $B$, and let $F(\xi) \rightarrow B$ be the bundle of complete flags. Corresponding to the $m$ canonical line bundles over $F(\xi)$ are $m$ classifying maps $\alpha_{i}: F(\xi) \rightarrow \mathbb{C} P^{\infty}$.

If $E^{*}$ is complex oriented, then a complex orientation $x \in E^{*}\left(\mathbb{C} P^{\infty}\right)$ determines $m$ classes $x_{i}=\alpha_{i}^{*}(x) \in E^{*}(F(\xi))$.

Proposition 2.4. (1) There is an identification

$$
E^{*}(F(\xi))=E^{*}(B)\left[x_{1}, \ldots, x_{m}\right] /\left(\sigma_{i}\left(\left\{x_{j}\right\}\right)-c_{i}(\xi)\right)
$$

where $\sigma_{i}$ is the $i^{\text {th }}$ elementary symmetric function.

(2) The $E^{*}(B)$-module $E^{*}(F(\xi))$ is free of rank $m$ !.

(3) If $\xi^{\prime}$ is the bundle over $B^{\prime}$ induced by a map $B^{\prime} \rightarrow B$, then the map

$$
E^{*}\left(B^{\prime}\right) \otimes_{E^{*}(B)} E^{*}(F(\xi)) \rightarrow E^{*}\left(F\left(\xi^{\prime}\right)\right)
$$

is an isomorphism.

(4) There are natural isomorphisms

$$
E^{*}(F(\xi)) \otimes_{E^{*}(B)} E^{*}(F(\xi)) \simeq E^{*}\left(F(\xi) \times_{B} F(\xi)\right) .
$$

Proof. Statement (1) is classical (e.g. compare with [Ati67, Prop.2.7.13]), and (2) follows as a matter of pure algebra. Then (3) follows from (2), and (4) is the special case of (3) applied to the map $F(\xi) \rightarrow B$ itself.

Proposition 2.5. The following sequence is an equalizer:

$$
E^{*}(B) \rightarrow E^{*}(F(\xi)) \rightrightarrows E^{*}\left(F(\xi) \times{ }_{B} F(\xi)\right) .
$$

Proof. We apply Proposition 2.3 to the following situation:

The category $\mathcal{C}$ is the category whose objects are pairs $(B, \xi)$ where $\xi$ is a complex vector bundle over $B$. A morphism from $\left(B^{\prime}, \xi^{\prime}\right)$ to $(B, \xi)$ consists of a map $f$ : $B^{\prime} \rightarrow B$, together with a bundle isomorphism $\xi^{\prime} \simeq f^{*}(\xi)$. The category $\mathcal{A}$ will be the category of $E^{*}$-modules.

$\mathcal{F}(B, \xi)$ will be the pair $\left(F(\xi), p^{*}(\xi)\right)$, where $p: F(\xi) \rightarrow B$ is the projection. $\mathcal{H}(B, \xi)=E^{*}(B)$.

The natural transformation ' $p$ ' of subsection 2.1 will be induced in the obvious way by $p: F(\xi) \rightarrow B$. To define ' $r$ ', first note that the composite

$$
E^{*}(B) \stackrel{p^{*}}{\longrightarrow} E^{*}(F(\xi)) \stackrel{\pi}{\longrightarrow} E^{*}(F(\xi)) /\left(x_{1}, \ldots, x_{m}\right)
$$

is an isomorphism by the previous proposition. We then define $r: E^{*}(F(\xi)) \rightarrow$ $E^{*}(B)$ to be the inverse of this natural isomorphism, precomposed with $\pi$. 
We end this subsection by noting that we can apply these results inductively to

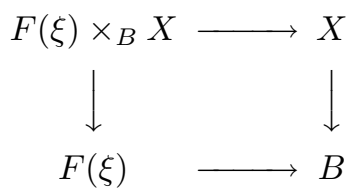

to get similar assertions with $F(\xi)$ replaced by an iterated fiber product of flag bundles, or more generally, a disjoint union of iterated fiber products of flag bundles.

2.3. Equivariant flag bundles. Now let $G$ be a finite group7

Proposition 2.6. Let $\xi$ be an $m$-dimensional equivariant complex vector bundle over a $G$-space $X$. Let $Y \rightarrow B$ be either the map

i) $E G \times{ }_{G} F(\xi) \rightarrow E G \times{ }_{G} X$, or

ii) $F(\xi)^{A} \rightarrow X^{A}$ where $A$ is an abelian subgroup of $G$.

Then, for any complex oriented theory $E^{*}$, we have

(1) The $E^{*}(B)$-module $E^{*}(Y)$ is free of rank $m$ !.

(2) The following sequence is an equalizer:

$$
E^{*}(B) \rightarrow E^{*}(Y) \rightrightarrows E^{*}\left(Y \times_{B} Y\right) .
$$

Proof. In case i), $Y$ is the bundle of complete flags in $E G \times_{G} \xi$, and the result follows from the previous subsection.

The more delicate case is ii). Note that we can assume that $G=A$ and $X=X^{A}$. Thus we need to analyze $F(\xi)^{A}$, where $\xi$ is an equivariant $n$-dimensional bundle over a trivial $A$-space $X$. Let $L_{1}, \ldots, L_{k}$ denote the distinct irreducible $A$-modules. In [Seg68:2 Prop.2.2], G. Segal noted that $\xi$ will admit a decomposition

$$
\xi \simeq \bigoplus_{i=1}^{k} L_{i} \otimes \xi_{i}
$$

for uniquely defined nonequivariant bundles $\xi_{i}$ over $X$.

Let $\xi_{i}$ have dimension $m_{i}$. Since $A$ is abelian, each of the $L_{i}$ is one dimensional, and thus $m_{1}+\cdots+m_{k}=m$. By inspection, it then follows that, as spaces over $X$,

$$
F(\xi)^{A} \simeq \coprod F\left(\xi_{1}\right) \times_{X} F\left(\xi_{2}\right) \times_{X} \cdots \times_{X} F\left(\xi_{k}\right)
$$

with the disjoint union running over all partitions of $m$ into $k$ subsets of cardinality $m_{1}, \ldots, m_{k}$. Noting that the number of such partitions is $\frac{m !}{m_{1} ! \cdots m_{k} !}$ and that

$$
\left(\frac{m !}{m_{1} ! \cdots m_{k} !}\right) m_{1} ! \cdots m_{k} !=m !
$$

the proposition now follows from the previous subsection.

The following consequence of our analysis is a generalization of the well-known fact that the Euler characteristic of $U(m) / T^{m}$ is $m$ !. It will be used in our proof of Theorem B.

Corollary 2.7. Let $\xi$ be an $m$-dimensional equivariant complex vector bundle over a finite $G-C W$ complex $X$. If $A$ is any abelian subgroup of $G$, then $\chi\left(F(\xi)^{A}\right)=$ $m ! \chi\left(X^{A}\right)$.

\footnotetext{
${ }^{7}$ More generally, the results and proofs of this subsection remain valid if $G$ is any compact Lie group.
} 
Finally, we will use without further comment:

Proposition 2.8. Let $\xi$ be an equivariant complex vector bundle over a finite $G-$ $C W$ complex $X$. Then $F(\xi)$ also has the homotopy type of a finite $G-C W$ complex.

Proof. By induction on the cells of $X$, we may assume that $X=G / H$ for some $H \leq G$. In this case $F(\xi)$ is a compact smooth $G$-manifold, and thus admits the structure of a $G-\mathrm{CW}$ complex (necessarily finite) by [Ill74].

Remark 2.9. Results in this section easily generalize to 'relative' versions involving pairs $X_{0} \subseteq X$ of $G$-spaces. This leads to the obvious relative versions of our main theorems.

\section{Rational equivariant stable homotopy and Artin's Theorem}

If $E$ is a generalized cohomology theory, one can regard the assignment

$$
X \longmapsto \tilde{E}^{*}\left(E G_{+} \wedge_{G} X\right)
$$

as an equivariant cohomology theory, defined on $\mathcal{C}_{G}$, the stable category of based finite $G-\mathrm{CW}$ complexes ([LMS86]). Although we make only elementary use of this deep fact, it is conceptually very illuminating. Here we begin with some general facts about rational equivariant cohomology theories 8 and then use the extra hypothesis that $E^{*}$ is complex oriented. Although many of these general facts are in the literature as parts of more general machines (see [LMS86, especially page 271], [Die79], and [Ara82]), we develop what we need from minimal prerequisites. For general background material on the stable category of finite $G-\mathrm{CW}$ complexes see also [Ada82] and [TtD:87.

3.1. Rational equivariant stable homotopy theory. Recall that the Burnside ring $A(G)$ is the Grothendieck group associated to the monoid of finite $G$-sets, with addition coming from disjoint union and multiplication from cartesian product9 Additively, $A(G)$ is the free abelian group on the isomorphism classes of transitive $G$-sets. Intuitively, $A(G)$ is the object containing the algebra of double coset formulae. There is an isomorphism of rings [TtD:87, $\S I I .8]$

$$
A(G) \simeq\left\{S^{0}, S^{0}\right\}_{G},
$$

where $\{X, Y\}_{G}$ denotes the stable equivariant homotopy group. Thus $A(G)$ acts on any equivariant cohomology theory, and, more generally, on any additive contravariant functor defined on $\mathcal{C}_{G}$.

There is a character theory for $A(G)$. Given $H<G$, let $\chi_{H}: A(G) \rightarrow \mathbb{Z}$ be defined by $\chi_{H}(S)=\left|S^{H}\right|$.

Lemma 3.1 ([Die79, page 3]). The map

$$
\prod_{(H)} \chi_{H}: A(G) \longrightarrow \prod_{(H)} \mathbb{Z}
$$

is an inclusion, and becomes an isomorphism after inverting $|G|$.

\footnotetext{
${ }^{8}$ What matters here is that the order of $G$ is inverted.

${ }^{9}$ The Burnside ring $A(G)$, used mainly in this section, should not be confused with the category $\mathcal{A}(G)$.
} 
Here, the product runs over the conjugacy classes $(H)$ of subgroups of $G$.

It follows that $1 \in A(G) \otimes \mathbb{Z}\left[|G|^{-1}\right]$ can be written as the sum of orthogonal idempotents

$$
1=\sum_{(H)} e_{H}
$$

where $\chi_{K}\left(e_{H}\right)$ is 1 if $K$ is conjugate to $H$, and 0 otherwise.

For an additive contravariant functor

$$
\tilde{h}: \mathcal{C}_{G} \longrightarrow \mathbb{Z}\left[|G|^{-1}\right] \text {-modules, }
$$

this decomposition gives a natural splitting

$$
\tilde{h}(X) \simeq \prod_{(H)} e_{H} \tilde{h}(X) .
$$

The following is a key observation, for which we would like to thank J. F. Adams.

Proposition 3.2. The natural $G-m a p$

$$
G / H_{+} \wedge X^{H} \rightarrow X
$$

induces an isomorphism

$$
e_{H} \tilde{h}(X) \simeq e_{H} \tilde{h}\left(G / H_{+} \wedge X^{H}\right)^{W_{G}(H)} .
$$

Here $Y_{+}$denotes the union of $Y$ with a disjoint basepoint, and the Weyl group $W_{G}(H)$ is the quotient of the normalizer $N_{G}(H)$ of $H$ in $G$ by $H$, acting on the right of $G / H_{+} \wedge X^{H}$ via $(g H, x) \cdot(n H)=\left(g n H, n^{-1} x\right)$.

We postpone the proof of Proposition 3.2 until the end of the section.

3.2. Complex oriented equivariant stable homotopy. Fix an embedding $G \subset$ $U(m)$, and let $F$ be the flag manifold $U(m) / T^{m}$. The main result of this section is the next theorem, which includes Theorem $\mathrm{A}$ as a special case.

Theorem 3.3. Let $h$ be a contravariant functor from the category of (unbased) $G-C W$ complexes to $\mathbb{Z}\left[|G|^{-1}\right]$-modules. Suppose that $h$ satisfies

(1) $h(X) \rightarrow h(X \times F)$ is a monomorphism for all $X$.

(2) There exists a contravariant additive functor

$$
\tilde{h}: \mathcal{C}_{G} \longrightarrow \mathbb{Z}\left[|G|^{-1}\right] \text {-modules }
$$

extending $h: \tilde{h}\left(X_{+}\right)=h(X)$.

Then, for all $G-C W$ complexes $X$, each of the maps

$$
h(X) \rightarrow \lim _{A \in \mathcal{A}(G)} h\left(G \times{ }_{A} X\right) \rightarrow \int_{A \in \mathcal{A}(G)} h\left(G / A \times X^{A}\right)
$$

is an isomorphism.

To see that Theorem Afollows, let

$$
h(X)=E^{*}\left(E G \times_{G} X\right) \otimes \mathbb{Z}\left[|G|^{-1}\right],
$$

with $E^{*}$ a complex oriented cohomology theory. Then Proposition 2.6 shows that condition (1) applies, and condition (2) holds by letting

$$
\tilde{h}(X)=\tilde{E}^{*}\left(E G_{+} \wedge_{G} X\right) \otimes \mathbb{Z}\left[|G|^{-1}\right] .
$$


Proof of Theorem 3.3. We begin by showing that, under the hypotheses on $h$, there are natural isomorphisms

$$
h(X) \simeq \prod_{(A)} e_{A} h\left(G / A \times X^{A}\right)^{W_{G}(A)}
$$

with the product running only over the conjugacy classes of abelian subgroups of $G$.

Since $h$ extends to $\tilde{h}$, there are natural commutative diagrams

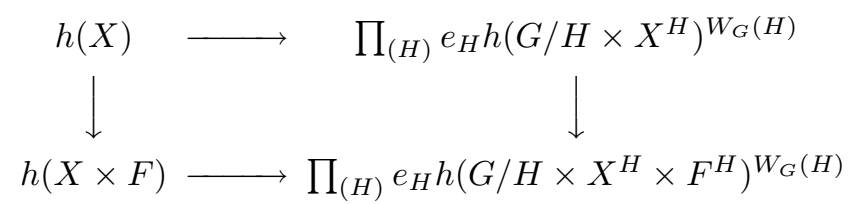

with both horizontal maps isomorphisms, and the products running over all conjugacy classes of subgroups. As the left vertical map is a monomorphism by assumption, so are each of the components of the right one. But $F^{H}=\emptyset$ unless $H$ is abelian, so we conclude that only the terms in these products corresponding to abelian subgroups will be nonzero.

Now we apply the method of Proposition 2.3 to the following two situations. In both cases, $\mathcal{C}$ will be the category of $G-\mathrm{CW}$ complexes, and $\mathcal{H}(X)=h(X)$. For $X$ a $G-\mathrm{CW}$ complex, let

$$
\mathcal{F}_{1}(X)=\coprod_{A \in \mathcal{A}(G)} G \times_{A} X
$$

and let

$$
\mathcal{F}_{2}(X)=\coprod_{A \in \mathcal{A}(G)} G / A \times X^{A}
$$

The apparent $G$-maps $\mathcal{F}_{2}(X) \rightarrow \mathcal{F}_{1}(X) \rightarrow X$ define ' $p$ ' in each case. To see that there exists a natural retraction $r(X): h\left(\mathcal{F}_{i}(X)\right) \rightarrow h(X)$ for $i=1,2$, we note that the isomorphism (3.4) will factor through $h(p(X))$ in each case.

Applying Proposition 2.3. we conclude that for $i=1,2$, we have equalizer diagrams

$$
h(X) \rightarrow h\left(\mathcal{F}_{i}(X)\right) \rightrightarrows h\left(\mathcal{F}_{i}\left(\mathcal{F}_{i}(X)\right)\right)
$$

To complete the proof of the theorem, one checks that when $i=1$ the equalizer of the two maps on the right is

$$
\lim _{\mathcal{A}(G)} h\left(G \times_{A} X\right),
$$

and when $i=2$ the equalizer is

$$
\int_{\mathcal{A}(G)} h\left(G / A \times X^{A}\right) .
$$

Remarks 3.5. (1) The standard properties of the transfer lead to an argument like the one above establishing (3.4) to show that for any cohomology theory $E, e_{H}$. $E^{*}\left(B H \times X^{H}\right)^{W_{G}(H)} \otimes \mathbb{Z}\left[|G|^{-1}\right]$ is zero unless $H$ is a $p$-group. (This is the effect of working with $A(G)$-modules whose action extends to $\hat{A}(G)$.) Thus the inverse limit in Theorem A need be taken only over abelian $p$-groups. 
(2) Note that our proof of Theorem A shows that the inverse limit is an $E^{*}$ module direct summand of the product. Thus $E^{*}(B G) \otimes \mathbb{Z}\left[|G|^{-1}\right]$ is a flat (or projective) $E^{*} \otimes \mathbb{Z}\left[|G|^{-1}\right]$-module for all $G$ if it is true for abelian $G$. For example, $M U^{*}(B G) \otimes \mathbb{Z}\left[|G|^{-1}\right]$ is thus a flat $M U^{*}$-module by Landweber's observation in Lan71.

3.3. Proof of Proposition 3.2. We couldn't quite find this in the literature. It seems likely that a combination of results in Chapter 5, Section 6 of [LMS86] would yield the proposition. The following proof is based on an unpublished argument of Adams.

Lemma 3.6. The fixed point map

$$
f_{X, Y}^{G}:\{X, Y\}_{G} \otimes \mathbb{Z}\left[|G|^{-1}\right] \longrightarrow \prod_{(H)}\left\{X^{H}, Y^{H}\right\}^{W_{G}(H)} \otimes \mathbb{Z}\left[|G|^{-1}\right]
$$

is an isomorphism for all $Y$ and all finite $G$-complexes $X$.

Here $\{X, Y\}_{G}$ denotes the equivariant stable maps, and $\left\{X^{H}, Y^{H}\right\}^{W_{G}(H)}$ denotes the $W_{G}(H)$-invariants of the $W_{G}(H)$-module $\left\{X^{H}, Y^{H}\right\}$ of nonequivariant maps.

Proof. Suppose first that $X=S^{n}$ and $Y=S^{m}$ with trivial $G$-action. Segal observed in Seg71 that

$$
\left\{S^{n}, S^{m}\right\}_{G}=\prod_{(H)} \pi_{n-m}^{S}\left(B W_{G}(H)_{+}\right) .
$$

Thus when $m=n$, the map $f_{S^{n}, S^{m}}^{G}$ reduces to the isomorphism of Lemma 3.1, and when $m \neq n$, both the domain and range of $f_{S^{n}, S^{m}}^{G}$ are 0 .

Next one observes that in each variable, both the domain and the range of $f_{X, Y}^{G}$ behave in the same way with respect to induction and $G$-cofibrations. For induction, we have that Ada82

$$
\left\{G_{+} \wedge_{H} X, Y\right\}_{G} \simeq\{X, Y\}_{H} \simeq\left\{X, G_{+} \wedge_{H} Y\right\}_{G},
$$

and, perhaps less obviously, that as $W_{G}(K)$-spaces

$$
\left(G_{+} \wedge_{H} X\right)^{K}=\coprod_{\left(K^{\prime}\right)} W_{G}\left(K^{\prime}\right)_{+} \wedge_{W_{H}\left(K^{\prime}\right)} X^{K^{\prime}}
$$

where the union is over conjugacy classes in $H$ of subgroups conjugate to $K$ in $G$. For cofibrations, note that if $X \rightarrow Y \rightarrow Z$ is a $G$-cofibration, then $X^{H} \rightarrow Y^{H} \rightarrow$ $Z^{H}$ is a $W_{G}(H)$-cofibration. The logic now goes as follows: $f_{S^{n}, S^{m}}^{G}$ is known to be an isomorphism for all $G, m$, and $n$. Thus $f_{G / H_{+} \wedge S^{n}, S^{m}}^{G}$ is an isomorphism for all $G, H \leq G, m$, and $n$. Thus $f_{X, S^{m}}^{G}$ is an isomorphism for all groups $G$, finite $G-$ complexes $X$, and $m$ (a 5-lemma argument). Thus $f_{X, G / H_{+} \wedge S_{m}^{m}}^{G}$ is an isomorphism for all $G, H \leq G$ and finite $G$ complexes $X$, and $m$. Thus $f_{X, Y}^{G}$ is an isomorphism for all $G$ and all finite $G$-complexes, $X$ and $Y$. Finally, an arbitrary $Y$ is the direct limit of its finite subcomplexes, and homotopy groups of maps from a finite complex commutes with direct limits, showing that $f_{X, Y}^{G}$ is an isomorphism in general.

Letting $X=Y$ in Lemma 3.6, we get a decomposition

$$
1_{X}=\sum_{(H)} e_{H, X} \in\{X, X\}_{G} \otimes \mathbb{Z}\left[|G|^{-1}\right] .
$$


That this notation is redundant is shown by

Lemma 3.7. $e_{H, X}=e_{H} \wedge 1_{X}$.

Proof. The point here is that if $f$ and $g$ are $G$-maps, then $(f \wedge g)^{K}=f^{K} \wedge g^{K}$. Thus $\left(e_{K} \wedge 1_{X}\right)^{K}=e_{H}^{K} \wedge 1_{X^{K}}$, which is zero if $K \notin(H)$ and the identity if $K=H$.

As a corollary of this we have

Lemma 3.8. Let $\tilde{h}$ be a functor as in Proposition 3.2 and let $f, g \in\{Y, Z\}_{G} \otimes$ $\mathbb{Z}\left[|G|^{-1}\right]$. If $f^{H} \simeq g^{H}$, then

$$
e_{H} f^{*}=e_{H} g^{*}: e_{H} \tilde{h}(Z) \rightarrow e_{H} \tilde{h}(Y)
$$

Lemma 3.9. With $\tilde{h}$ as in Proposition 3.2, the map

$$
\pi \wedge 1: G / H_{+} \wedge X \rightarrow X
$$

induces an isomorphism

$$
e_{H} \tilde{h}(X) \simeq e_{H} \tilde{h}\left(G / H_{+} \wedge X\right)^{W_{G}(H)} .
$$

Proof. Repressing $\otimes \mathbb{Z}\left[|G|^{-1}\right]$ from the notation, let $t \in\left\{S^{0}, G / H_{+}\right\}_{G}$ be any map such that $t^{H} \in\left\{S^{0}, W_{G}(H)_{+}\right\}$is the sum of the maps sending the nonbasepoint of $S^{0}$ to the points of $(G / H)^{H}=W_{G}(H)$. Then $(\pi \circ t)^{H}=\left|W_{G}(H)\right| \in\left\{S^{0}, S^{0}\right\}$ and $(t \circ \pi)^{H}=\sum_{w \in W_{G}(H)} w \in\left\{W_{G}(H)_{+}, W_{G}(H)_{+}\right\}$. Hence the preceding lemma implies that $\pi \wedge 1$ and $t \wedge 1$ induce natural maps

$$
e_{H} \tilde{h}(X) \rightleftarrows e_{H} \tilde{h}\left(G / H_{+} \wedge X\right)
$$

satisfying the same formulae. The lemma follows.

Proof of Proposition 3.2. The map $G / H_{+} \wedge X^{H} \rightarrow X$, sending $(g H, x)$ to $g x$, factors as

$$
G / H_{+} \wedge X^{H} \stackrel{j}{\longrightarrow} G / H_{+} \wedge X \stackrel{\pi \wedge 1}{\longrightarrow} X
$$

where $j(g H, x)=(g H, g x)$. Note that $j$ is a map of left $G$-spaces, and is a map of right $W_{G}(H)$-spaces if $W_{G}(H)$ acts on $G / H_{+} \wedge X^{H}$ by

$$
(g H, x) \cdot(n H)=\left(g n H, n^{-1} x\right) .
$$

On $H$-fixed point sets, $j^{H}: W_{G}(H)_{+} \wedge X^{H} \rightarrow W_{G}(H)_{+} \wedge X^{H}$ is the homeomorphism $(w, x) \mapsto(w, w x)$. Thus we can apply the last two lemmas to conclude that $j$ and $\pi \wedge 1$ induce isomorphisms

$$
e_{H} h(X) \stackrel{\simeq}{\longrightarrow} e_{H} h\left(G / H_{+} \wedge X\right)^{W_{G}(H)} \stackrel{\simeq}{\longrightarrow} e_{H} h\left(G / H_{+} \wedge X^{H}\right)^{W_{G}(H)},
$$

as needed.

\section{Complex oriented Euler Characteristics}

Let $X$ be a finite $G$-complex. In this section we prove Theorem B: the Morava $\mathrm{K}$-theory Euler characteristic

$$
\chi_{n, p}^{G}(X)=\operatorname{dim} K(n)^{\text {even }}\left(E G \times_{G} X\right)-\operatorname{dim} K(n)^{\text {odd }}\left(E G \times_{G} X\right)
$$


will be expressed in terms of the ordinary Euler characteristics of the fixed point spaces $X^{A}$, with $A \leq G$ abelian. When $X$ is a point, our computation specializes to show that $\chi_{n, p}^{G}$, the Euler characteristic of $K(n)^{*}(B G)$, equals

$$
\sum_{A \leq G} \frac{|A|}{|G|}\left|A_{(p)}\right|^{n} \mu_{\mathcal{A}(G)}(A)
$$

where, for all abelian subgroups $A \leq G$, the integer $\mu_{\mathcal{A}(G)}(A)$ is defined by downward induction on $A \in \mathcal{A}(G)$ by the equation

$$
\sum_{\substack{A \leq B \\ B \text { abelian }}} \mu_{\mathcal{A}(G)}(B)=1 .
$$

We then use elementary group theory to show that this sum equals the number of $G$-orbits in $G_{n, p}$.

4.1. Additive functions. Let $M$ be an abelian group.

Definition 4.1 (Compare with [TtD:87, p. 227]). A function

$$
X \mapsto \chi(X) \in M
$$

associating to each finite $G-\mathrm{CW}$ complex $X$, an element of $M$ is additive if it satisfies the following conditions:

(1) If $X$ and $Y$ are $G$-homotopy equivalent, then $\chi(X)=\chi(Y)$.

(2) If $Z=X \cup Y$ and $W=X \cap Y$, then

$$
\chi(W)+\chi(Z)=\chi(X)+\chi(Y) .
$$

(3) $\chi(\phi)=0$.

Since any finite $G-\mathrm{CW}$ complex can be built using cells of the form $G / H \times D^{n}$, we have

Lemma 4.2. An additive function $\chi$ is determined by the values it takes on the $G$-sets $G / H$ for all subgroups $H \leq G$.

A slightly more refined statement is

Lemma 4.3. The function which associates to each finite $G$-set $X$, the class of $X$ in the Burnside ring $A(G)$, extends to a unique additive function $\chi_{u n i v}$. This additive function is universal in the sense that

$$
\begin{aligned}
\operatorname{Hom}(A(G), M) & \rightarrow\left\{\begin{array}{c}
\text { additive functions with } \\
\text { values in } M
\end{array}\right\} \\
f & \mapsto f \circ \chi_{\text {univ }}
\end{aligned}
$$

is a bijection.

From now on, unless otherwise stated, additive functions will take values in the abelian group $\mathbb{Z}\left[|G|^{-1}\right]$.

Example/Definition 4.4. i) For a subgroup $K \leq G$, let $\chi_{K}(X)$ be the Euler characteristic of the fixed point space $X^{K}$. If $K^{\prime}$ is conjugate to $K$, then $\chi_{K}=\chi_{K^{\prime}}$.

ii) Let $\mu_{H}$ be the additive function defined by downward induction on $H$ by

$$
\sum_{K \leq H} \mu_{H}=\chi_{K} .
$$

This also depends only on the conjugacy class of $H$. 
We remark that in the proof of Theorem 4.8 below, we will use that the additive functions $\chi_{K}$ are also multiplicative:

$$
\chi_{K}(X \times Y)=\chi_{K}(X) \chi_{K}(Y) .
$$

Lemma 4.5. Let $K$ run through a set of representatives for the conjugacy classes of subgroups of $G$. Then each of the sets

$$
\left\{\chi_{K}\right\} \quad \text { and } \quad\left\{\mu_{K}\right\}
$$

is a basis of the $\mathbb{Z}\left[|G|^{-1}\right]$-module of additive functions.

Proof. To show that the set $\left\{\chi_{K}\right\}$ is a basis amounts, by Lemma 4.3 to showing that the homomorphisms

$$
\begin{aligned}
A(G) & \rightarrow \mathbb{Z}\left[|G|^{-1}\right] \\
X & \mapsto\left|X^{K}\right|
\end{aligned}
$$

form a basis of

$$
\operatorname{Hom}\left(A(G), \mathbb{Z}\left[|G|^{-1}\right]\right) \text {. }
$$

This is precisely the content of Lemma 3.1 The assertion that $\left\{\mu_{K}\right\}$ is a basis now follows easily from the definition.

Any additive function $\chi$ can thus be written as a linear combination of the $\mu_{H}$, with coefficients which depend only on the values $\chi(G / H)$. The next proposition makes this more precise.

Proposition 4.6. If $\chi$ is an additive function, then

$$
\begin{aligned}
\chi & =\sum_{(H)} \frac{1}{\left|W_{G}(H)\right|} \chi(G / H) \mu_{H} \\
& =\frac{1}{|G|} \sum_{H}|H| \chi(G / H) \mu_{H} .
\end{aligned}
$$

Proof. By linearity we need only check the first equality when $\chi=\chi_{K}$, which is easy to do. First note that

$$
\chi_{K}(G / H)=\left|(G / H)^{K}\right|=\sum_{\substack{H^{\prime} \geq K \\ H^{\prime} \sim H}}\left|W_{G}(H)\right|,
$$

thus

$$
\begin{aligned}
\sum_{(H)} \frac{1}{\left|W_{G}(H)\right|}\left|(G / H)^{K}\right| \mu_{H} & =\sum_{(H)} \sum_{\substack{K \leq H^{\prime} \\
H^{\prime} \in(H)}} \mu_{H} \\
& =\sum_{K \leq H} \mu_{H} \\
& =\chi_{K}
\end{aligned}
$$

as needed. For the second equality, recall that $|G| /\left|N_{G}(H)\right|$ is the number of $H^{\prime}$ conjugate to $H$. 
4.2. Complex oriented additive functions. Recall that, if $\xi$ is a complex vector bundle over $X, F(\xi)$ denotes the associated bundle of complete flags. The following definition is motivated by complex oriented descent.

Definition 4.7. An additive function $\chi$ is complex oriented if for every $n$-dimensional equivariant complex vector bundle $\xi$ over $X$,

$$
\chi(F(\xi))=n ! \cdot \chi(X) .
$$

Our complex oriented version of Lemma 4.5 is

Theorem 4.8. An additive function $\chi$ is complex oriented if and only if it is a linear combination of the functions $\chi_{A}$ with $A$ abelian.

Proof. We first note that Corollary 2.7 precisely says that if $A \leq G$ is abelian, then $\chi_{A}$ is complex oriented.

For the converse, suppose that $\chi=\sum_{H} a_{H} \chi_{H}$ is complex oriented. Choose an $n$-dimensional faithful representation $V$. Since the representation is faithful, each isotropy subgroup in the $G$-space $F(V)$ will be abelian. Thus if $H \leq G$ is nonabelian, then $F(V)^{H}=\phi$ and so $\chi_{H}(F(V))=0$. We then compute, for all $X$,

$$
\begin{aligned}
n ! \sum_{H} a_{H} \chi_{H}(X) & =n ! \chi(X) \\
& =\chi(X \times F(V)) \\
& =\sum_{H} a_{H} \chi_{H}(X \times F(V)) \\
& =\sum_{H} a_{H} \chi_{H}(X) \chi_{H}(F(V)) \\
& =n ! \sum_{A} a_{A} \chi_{A}(X),
\end{aligned}
$$

where the last sum is only over abelian subgroups. It follows from the linear independence of the $\chi_{H}$ that $a_{H}=0$ if $H$ is not abelian.

Now we will prove an analogue of Proposition 4.6

Definition 4.9. For an abelian subgroup $A \leq G$, the additive function $\mu_{A}^{\mathbb{C}}$ is defined by downward induction on $A$ by

$$
\sum_{\substack{A \leq B \\ B \text { abelian }}} \mu_{B}^{\mathbb{C}}=\chi_{A} .
$$

In particular $\mu_{A}^{\mathbb{C}}$ (pt.) $=\mu_{\mathcal{A}(G)}(A)$, the Moebius function of the beginning of this section.

Proposition 4.10. If $\chi$ is a complex oriented additive function, then

$$
\chi=\frac{1}{|G|} \sum_{A}|A| \chi(G / A) \mu_{A}^{\mathbb{C}} .
$$

Proof. Observe that if $Y$ is a $G$-space with all isotropy groups abelian, then

$$
\mu_{H}(Y)= \begin{cases}\mu_{H}^{\mathbb{C}}(Y) & \text { if } H \text { is abelian, } \\ 0 & \text { otherwise }\end{cases}
$$


We apply this to the case $Y=X \times F(V)$, where $V$ is a faithful $n$-dimensional representation. Using 4.6, we have, for all $X$,

$$
\begin{aligned}
n ! \chi(X) & =\chi(X \times F(V)) \\
& =\frac{1}{|G|} \sum_{H}|H| \chi(G / H) \mu_{H}(X \times F(V)) \\
& =\frac{1}{|G|} \sum_{A}|A| \chi(G / A) \mu_{A}^{\mathbb{C}}(X \times F(V)) \\
& =\frac{n !}{|G|} \sum_{A}|A| \chi(G / A) \mu_{A}^{\mathbb{C}}(X) .
\end{aligned}
$$

\subsection{Morava K-theory Euler characteristics.}

Proposition 4.11 (see also [Rav82]). If $X$ is a finite $G-C W$ complex, then

$$
K(n)^{*}\left(E G \times_{G} X\right)
$$

is a finite dimensional vector space over $K(n)^{*}$. Thus $\chi_{n, p}^{G}(X)$ is a complex oriented additive function. Moreover, for an abelian subgroup $A \leq G$, with $p$-Sylow subgroup $A_{(p)}$

$$
\chi_{n, p}^{G}(G / A)=\left|A_{(p)}\right|^{n} .
$$

Proof. By replacing $X$ with $X \times F(V)$ and using complex oriented descent, it can be assumed that the cells of $X$ are of the form $G / A \times D^{k}$ with $A$ abelian. An induction over the skeleta reduces to the case $X=G / A$. But

$$
K(n)^{*}\left(E G \times{ }_{G} G / A\right)=K(n)^{*}(B A),
$$

so the result follows from the well-known calculation of $K(n)^{*}(B A)$. (See Corollary 5.10 below.)

Proposition 4.10 immediately implies the next theorem, which was also stated as Theorem B (Part 2) in the introduction.

Theorem 4.12. For any finite $G-C W$ complex $X$,

$$
\chi_{n, p}^{G}(X)=\frac{1}{|G|} \sum_{A}|A|\left|A_{(p)}\right|^{n} \mu_{A}^{\mathbb{C}}(X)
$$

the sum being over the set of abelian subgroups $A \leq G$. In particular (taking $X$ to be a point),

$$
\chi_{n, p}^{G}=\frac{1}{|G|} \sum_{A}|A|\left|A_{(p)}\right|^{n} \mu_{\mathcal{A}(G)}(A) .
$$

Now we count the $G$-orbits in $G_{n, p}=\operatorname{Hom}\left(\mathbb{Z}_{p}^{n}, G\right)$. For convenience, let $\Lambda=\mathbb{Z}_{p}^{n}$.

Lemma 4.13. $|\operatorname{Hom}(\Lambda, G) / G|=\frac{1}{|G|}|\operatorname{Hom}(\mathbb{Z} \times \Lambda, G)|$.

Proof. After choosing a generator of $\mathbb{Z}$, the fiber of the restriction mapping

$$
\operatorname{Hom}(\mathbb{Z} \times \Lambda, G) \rightarrow \operatorname{Hom}(\Lambda, G)
$$


over a map $\alpha$ can be identified with the centralizer, $C_{G}(\alpha)$, of the image of $\alpha$. Thus

$$
\begin{aligned}
|\operatorname{Hom}(\mathbb{Z} \times \Lambda, G)| & =\sum_{\alpha: \Lambda \rightarrow G}\left|C_{G}(\alpha)\right| \\
& =|G| \sum_{\alpha: \Lambda \rightarrow G}\left(\frac{\left|C_{G}(\alpha)\right|}{|G|}\right) \\
& =|G| \cdot|\operatorname{Hom}(\Lambda, G) / G| .
\end{aligned}
$$

The last equality comes from the isomorphism of $G$-sets:

$$
\operatorname{Hom}(\Lambda, G) \simeq \coprod_{\alpha \in \operatorname{Hom}(\Lambda, G) / G} G / C_{G}(\alpha) .
$$

Lemma 4.14. There is an equality

$$
|\operatorname{Hom}(\mathbb{Z} \times \Lambda, G)|=\sum_{A}|\operatorname{Hom}(\mathbb{Z} \times \Lambda, A)| \mu_{\mathcal{A}(G)}(A) .
$$

Proof. Note that the image of $\alpha \in \operatorname{Hom}(\mathbb{Z} \times \Lambda, G)$ is abelian. Thus

$$
\begin{aligned}
|\operatorname{Hom}(\mathbb{Z} \times \Lambda, G)| & =\sum_{\alpha} 1 \\
& =\sum_{\alpha} \sum_{\operatorname{Image}(\alpha) \leq A} \mu_{\mathcal{A}(G)}(A) \\
& =\sum_{A} \sum_{\alpha} \mu_{\mathcal{A}(G)}(A) \\
& =\sum_{A} \mid \operatorname{Hom}(\alpha) \leq A
\end{aligned}
$$

Now note that if $A$ is abelian,

$$
\left|\operatorname{Hom}\left(\mathbb{Z} \times \mathbb{Z}_{p}^{n}, A\right)\right|=|A| \cdot\left|A_{(p)}\right|^{n} .
$$

Thus the last two lemmas combine to yield

Corollary 4.15. $\left|\operatorname{Hom}\left(\mathbb{Z}_{p}^{n}, G\right) / G\right|=\sum_{A} \frac{|A|}{|G|}\left|A_{(p)}\right|^{n} \mu_{\mathcal{A}(G)}(A)$.

This formula, together with Theorem 4.12 yields Theorem B (Part 1).

$$
\text { 5. Formal groups And } E^{*}(B A)
$$

5.1. Formal groups and their height. The complex orientation of $E$ determines a formal group law $F$ over $E^{*}$ : the orientation

$$
x \in E^{2}\left(\mathbb{C} P^{\infty}\right)
$$

gives rise to isomorphisms

$$
\begin{aligned}
E^{*}\left(\mathbb{C} P^{\infty}\right) & =E^{*}[[x], \\
E^{*}\left(\mathbb{C} P^{\infty} \times \mathbb{C} P^{\infty}\right) & =E^{*}\left[\left[x_{1}, x_{2}\right],\right.
\end{aligned}
$$


and the formal sum is the image of $x$ under the map classifying the tensor product of line bundles:

$$
\begin{aligned}
x_{1}+x_{2}=F\left(x_{1}, x_{2}\right) & =\mu^{*}(x) \in E^{*}\left[\left[x_{1}, x_{2}\right]\right], \\
\mu: \mathbb{C} P^{\infty} \times \mathbb{C} P^{\infty} & \rightarrow \mathbb{C} P^{\infty} .
\end{aligned}
$$

In algebro-geometric terms, the orientation $x$ is a coordinate on $F$. In ordinary geometric terms, the orientation is the first Chern class of a line bundle, and the formal group law is the formula for the first Chern class of a tensor product of line bundles.

We recall some standard notation Rav86. Given an integer $m$, the $m$-series of $F$ is the formal power series

$$
[m](x)=\overbrace{x+\ldots+\ldots}^{+\ldots} \in E^{*} \llbracket \llbracket x \rrbracket .
$$

The $m-$ series is an endomorphism of $F$ :

$$
[m](x+\underset{F}{+} y)=[m](x) \underset{F}{+}[m](y) .
$$

More generally, if $E^{*}$ is a complete, local ring with maximal ideal $\mathfrak{m}$, and a prime $p$ is in $\mathfrak{m}$, then, by continuity, one can define $[m](x) \in E^{*}[[x]]$ for any $m \in \mathbb{Z}_{p}$, and thus $\mathbb{Z}_{p}$ acts on $F$ as a ring of endomorphisms.

Suppose we are in this last situation, so that the $\bmod \mathfrak{m}$ reduction, $F_{0}$, of $F$ is a formal group over a (graded) field of characteristic $p>0$. Then $F_{0}$ is more or less determined by a single invariant, its height (see e.g. [Rav86 Thm.A.2.2.11]). The height of $F_{0}$ is the degree of the isogeny "multiplication by $p$ ", and can be defined as follows. If the $p$-series of $F_{0}$ is identically 0 , we say that the height is $\infty$. Otherwise, $[p](x)$ can be written uniquely in the form

$$
\begin{aligned}
{[p]_{F_{0}}(x) } & =f\left(x^{p^{n}}\right), \\
f^{\prime}(0) & \neq 0 \in E^{*} / \mathfrak{m},
\end{aligned}
$$

for some $n<\infty$, and we define the height of $F_{0}$ to be $n$.

From now on it will be assumed that the height of $F_{0}$ is an integer $n<\infty$.

5.2. The structure of $E^{*}[[x]] /\left(\left[p^{r}\right](x)\right)$. Fundamental to our work is an understanding of the $E^{*}$-algebra $E^{*}[[x]] /\left(\left[p^{r}\right](x)\right)$. We begin with a very general lemma, a form of the Weierstrass Preparation Theorem.

Lemma 5.1. Let $R$ be a graded commutative ring, complete in the topology defined by the powers of an ideal I. Suppose $\alpha(x) \in R[x]]$ satisfies $\alpha(x) \equiv u x^{d} \bmod$ $\left(I, x^{d+1}\right)$ with $u \in R$ a unit. Then

i) (Euclidean algorithm) Given $f(x) \in R \llbracket x \rrbracket$, there exist unique

$$
p(x) \in R[x] \text { and } q(x) \in R[[x]]
$$

such that

$$
f(x)=p(x)+\alpha(x) q(x)
$$

with $\operatorname{deg} p(x) \leq d-1$.

ii) The ring $R \llbracket x \rrbracket /(\alpha(x))$ is a free $R$-module with basis

$$
\left\{1, x, \ldots, x^{d-1}\right\} \text {. }
$$


iii) (Factorization) There is a unique factorization

$$
\alpha(x)=\epsilon(x) g(x)
$$

with $\epsilon(x)$ a unit and $g(x)$ a monic polynomial of degree $d$.

The number $d$ is called the Weierstrass degree of $g(x)$.

Proof. Statement (iii) follows easily from (ii). This in turn follows from (i), which is well known; see e.g. Lan78, pages 129-131]. (The assumption made there that $R$ is local is not used in the proof.)

We apply this to $\left[p^{r}\right](x) \in E^{*}[[x]]$. Our assumption on the height is that

$$
[p](x) \equiv u x^{p^{n}} \bmod \left(\mathfrak{m}, x^{p^{n}+1}\right)
$$

with $u \in E^{*}$ a unit 10 which implies that, for all $r$,

$$
\left[p^{r}\right](x) \equiv u_{r} x^{p^{r n}} \bmod \left(\mathfrak{m}, x^{p^{r n}+1}\right)
$$

where $u_{r}$ is a power of $u$ (depending on $r$ ), and thus a unit in $E^{*}$.

Proposition 5.2. $E^{*}[[x]] /\left(\left[p^{r}\right](x)\right)$ is a free $E^{*}$-algebra, with basis

$$
\left\{1, x, \ldots, x^{p^{r n}-1}\right\} .
$$

The formal group law induces a cocommutative coproduct on $E^{*}[[x]] /\left(\left[p^{r}\right](x)\right)$, making $E^{*}[[x]] /\left(\left[p^{r}\right](x)\right)$ into a Hopf algebra over $E^{*}$.

Proof. The first statement follows immediately from the previous lemma. The second then follows: note that

$$
E^{*}\left[\left[x_{1}\right]\right] /\left(\left[p^{r}\right]\left(x_{1}\right)\right) \otimes_{E^{*}} E^{*}\left[\left[x_{2}\right]\right] /\left(\left[p^{r}\right]\left(x_{2}\right)\right) \rightarrow E^{*}\left[\left[x_{1}, x_{2}\right]\right] /\left(\left[p^{r}\right]\left(x_{1}\right),\left[p^{r}\right]\left(x_{2}\right)\right)
$$

is an isomorphism. Thus we can define the coproduct

$$
E^{*}[[x]] /\left(\left[p^{r}\right](x)\right) \rightarrow E^{*}[[x]] /\left(\left[p^{r}\right](x)\right) \otimes_{E^{*}} E^{*}[[x]] /\left(\left[p^{r}\right](x)\right)
$$

to be the map corresponding to the formal sum.

There is a inverse system of Hopf algebras over $E^{*}$

$$
\cdots \rightarrow E^{*}[[x]] /\left(\left[p^{r+1}\right](x)\right) \rightarrow E^{*}[[x]] /\left(\left[p^{r}\right](x)\right) \rightarrow \cdots
$$

induced by sending $x \in E^{*}[[x]] /\left(\left[p^{r+1}\right](x)\right)$ to $x \in E^{*}[[x]] /\left(\left[p^{r+1}\right](x)\right)$, and a direct system of Hopf algebras over $E^{*}$

$$
\cdots \rightarrow E^{*}[[x]] /\left(\left[p^{r}\right](x)\right) \rightarrow E^{*}[[x]] /\left(\left[p^{r+1}\right](x)\right) \rightarrow \cdots
$$

induced by sending $x \in E^{*}[[x]] /\left(\left[p^{r}\right](x)\right)$ to $[p](x) \in E^{*}[[x]] /\left(\left[p^{r+1}\right](x)\right)$.

\footnotetext{
${ }^{10}$ In our graded setting, this unit $u$ will have degree $2-2 p^{n}$. If $E^{*}$ came equipped with a $B P^{*}$ orientation, then $u$ will be the image of $v_{n} \in B P^{*}$, thus one refers to $E^{*}$ as ' $v_{n}$-periodic'.
} 


\subsection{The group of torsion points.}

Definition 5.5. Given a graded $E^{*}$-algebra $R$ and integer $r \geq 0$, let

$$
p^{r} F(R)
$$

be the group of $E^{*}$-algebra homomorphisms

$$
E^{*}[[x]] /\left(\left[p^{r}\right](x)\right) \rightarrow R
$$

where the group structure is induced by the coalgebra structure on $E^{*}[[x]] /\left(\left[p^{r}\right](x)\right)$. The inverse system (5.3) induces a direct system of inclusions

$$
\cdots \rightarrow{ }^{r} F(R) \rightarrow p^{r+1} F(R) \rightarrow \cdots,
$$

and we let $p^{\infty} F(R)$ denote the colimit. We will regard $p^{\infty} F(R)$ as a subset of $R$ via evaluation on $x$.

Classically, the group $p_{\infty} F(R)$ arises in the following way. Given a local homomorphism

$$
E^{*} \rightarrow R
$$

of complete, graded, local rings, let $F(R)$ be the group whose underlying set is the set of homogeneous elements of degree 2 in the maximal ideal of $R$, and whose sum is the formal sum $x+{ }_{F} y$. In this case, the group $p^{r} F(R)$ will just be the subgroup of $F(R)$ consisting of elements killed by $p^{r}$, and $p^{\infty} F(R)$ will be the torsion subgroup 11 The point of our definition is that one can define $p^{\infty} F(R)$ for an arbitrary $E^{*}$-algebra $R$.

The following theorem is a variation of a theorem of Lubin and Tate [LT65].

Theorem 5.6. Let $L$ be an $E^{*}$-algebra that is also an algebraically closed graded field of characteristic 0 . Then

i) For each $r>0$, the group $p^{r} F(L)$ is isomorphic to $\left(\mathbb{Z} / p^{r}\right)^{n}$.

ii) The group $p^{\infty} F(L)$ is isomorphic to $\left(\mathbb{Q}_{p} / \mathbb{Z}_{p}\right)^{n}$.

Proof. By the definition of height,

$$
\left[p^{r}\right](x) \equiv \epsilon x^{p^{r n}} \bmod \left(\mathfrak{m}, x^{p^{r n}+1}\right), \quad \epsilon \text { a unit. }
$$

The elements of $p^{r} F(L)$ are the roots of $\left[p^{r}\right](x)$ in $L$. By [5.1 there are $p^{r n}$ of these roots, counted with multiplicity. The proof will be finished if we show that all these multiplicities are 1, for then $p^{r} F(L)$ will be an abelian $p$-group with exactly $p^{r n}$ distinct elements of order dividing $p^{r}$.

To show the multiplicities are 1 , we will show that $\left[p^{r}\right]^{\prime}(x)$ has no zeros in $L$. We need to introduce the logarithm of $F$ Haz78, Rav86. This is the unique power series

$$
\log _{F}(x) \in L[[x]]
$$

satisfying

$$
\begin{aligned}
\log _{F}(x) & =x+\cdots \\
\log _{F}(x+F y) & =\log _{F}(x)+\log _{F}(y)
\end{aligned}
$$

\footnotetext{
${ }^{11} \mathrm{As} \mathbb{Z}_{p}$ acts as a ring of endomorphisms on $F(R)$, the torsion in $F(R)$ will all be $p$-torsion.
} 
If $x$ is given degree 2, the logarithm of $F$ is homogeneous of degree 2. Note that the derivative of the logarithm has the form

$$
\log _{F}^{\prime}(x)=1+\cdots
$$

and hence is a unit in $L[[x]]$.

Taking the derivative of

$$
\log _{F}\left(\left[p^{r}\right](x)\right)=p^{r} \log _{F}(x)
$$

gives

$$
\log _{F}^{\prime}\left(\left[p^{r}\right](x)\right) \cdot\left[p^{r}\right]^{\prime}(x)=p^{r} \log _{F}^{\prime}(x),
$$

from which it follows that

$$
\left[p^{r}\right]^{\prime}(x)=p^{r} \cdot(\text { a unit in } L[[x])
$$

has no zeros, since $L$ has characteristic 0 . This completes the proof.

5.4. Cohomology of abelian groups. Much of the material in this section can be found in [RW80].

For a finite (or profinite) abelian group $A$, let

$$
A^{*}=\operatorname{Hom}\left(A, S^{1}\right)
$$

be the character group.

Lemma 5.7. Suppose that $A$ is cyclic of order $m$, and let

$$
x \in E^{2}(B A)
$$

be the first Chern class of a generator of the character group $A^{*}$. The ring

$$
E^{*}(B A)
$$

is isomorphic to

$$
E^{*}[[x] / /([m](x)) .
$$

Proof. The Gysin sequence of the fibration

$$
S^{1} \rightarrow B A \rightarrow \mathbb{C} P^{\infty}
$$

is a long exact sequence

$$
\cdots \rightarrow E^{*}[[x]] \stackrel{\cdot[m](x)}{\longrightarrow} E^{*}[[x]] \rightarrow E^{*} B A \rightarrow \cdots .
$$

Lemma 5.1 and our standing assumption that $E^{*}$ is complete imply that $[m](x)$ is not a zero divisor.

Corollary 5.8. Write $m=s p^{r}$, with $(s, p)=1$. The $E^{*}$-module $E^{*}(B \mathbb{Z} / m)$ is free of rank $p^{r n}$.

Proof. This follows from Proposition [5.2.

Lemma 5.9. Let $Y$ be a space with the property that $E^{*}(Y)$ is a finitely generated free module over $E^{*}$. Then for any space $X$, the map

$$
E^{*}(Y) \otimes_{E^{*}} E^{*}(X) \rightarrow E^{*}(Y \times X)
$$

is an isomorphism. 
Proof. Think of the map in question as a transformation of functors of $X$. Both sides convert pushout squares into Mayer-Vietoris sequences, and both sides convert infinite wedges into products. Since both functors agree when $X$ is a point, the transformation is an isomorphism.

Corollary 5.10. Suppose that $A$ is a finite abelian group with $p$-Sylow subgroup $A_{(p)}$. Then the $E^{*}$-module $E^{*}(B A)$ is free of rank $\left|A_{(p)}\right|^{n}$.

Proof. Write $A$ as a product of cyclic groups, and apply Corollary 5.8 and Lemma 5.9 .

Corollary 5.11. If $A$ is a finite abelian group, and $X$ is any space, then the map

$$
E^{*}(B A) \otimes_{E^{*}} E^{*}(X) \rightarrow E^{*}(B A \times X)
$$

is an isomorphism.

We end this section with a natural description of the spectrum of $E^{*}(B A)$.

Proposition 5.12. Let $A$ be a finite abelian group and $R$ an $E^{*-a l g e b r a . ~ T h e ~}$ natural transformation (of functors of pairs $(A, R)$ )

$$
\operatorname{Hom}_{E^{*}-\text { alg }}\left(E^{*}(B A), R\right) \rightarrow \operatorname{Hom}\left(A^{*}, p^{\infty} F(R)\right)
$$

defined by

$$
f \longmapsto\left\{\chi \mapsto f\left(c_{1}(\chi)\right)\right\}
$$

is an isomorphism.

Proof. First suppose that $A$ is cyclic of order $m$. If $m$ is prime to $p$, both the domain and range have one element. If $m=p^{r}$, choose a generator $\chi$ of $A^{*}$, and let $x \in E^{*}(B A)$ be the first Chern class of $\chi$. By Lemma [5.7] $E^{*}(B A)$ can then be identified with $E^{*}[[x]] /\left(\left[p^{r}\right](x)\right)$. Define a map

$$
\operatorname{Hom}\left(A^{*},{ }_{p^{r}} F(R)\right) \rightarrow \operatorname{Hom}_{E^{*} \text {-alg }}\left(E^{*}(B A), R\right)
$$

by

$$
g \longmapsto g(\chi)
$$

This is easily checked to be an inverse to (5.13). The result for arbitrary finite $A$ now follows since both sides of (5.13) convert finite sums of abelian groups into finite products.

Note that, under the correspondence of the proposition, the identity map on $E^{*}(B A)$ corresponds to a group homomorphism

$$
\phi_{\text {univ }}: A^{*} \rightarrow{ }_{p^{\infty}} F\left(E^{*}(B A)\right) \text {. }
$$

\section{Generalized characters}

In this section, we begin to relate group cohomology to certain rings of functions, ultimately proving the basic properties of $L\left(E^{*}\right)$ and related rings $L_{r}\left(E^{*}\right)$, where $L\left(E^{*}\right)$ is the $E^{*}$-algebra defined in the introduction. The proof of Theorem $\mathbb{C}$ then quite easily follows.

Let $\Lambda=\mathbb{Z}_{p}^{n}$, with quotient groups $\Lambda_{r}=\left(\mathbb{Z} / p^{r}\right)^{n}$. Then $\Lambda^{*} \simeq\left(\mathbb{Q}_{p} / \mathbb{Z}_{p}\right)^{n}$, and $\Lambda_{r}^{*} \simeq\left(\mathbb{Z} / p^{r}\right)^{n}$ is the subgroup of $\Lambda^{*}$ of elements of order $p^{r}$. 
6.1. Hopf algebra isomorphisms and a discriminant calculation. If $A$ is a finite abelian group and $R$ is an $E^{*}$-algebra, then $R^{A}$, the ring of $R$-valued functions on $A$, will be a Hopf algebra (over $E^{*}$ ). The next lemma will be used to construct maps to Hopf algebras of this elementary form, in particular to $R^{\Lambda_{r}^{*}}$.

Lemma 6.1. Let $A$ be a finite abelian group, and $R$ a graded $E^{*}$-algebra. The set of homomorphisms

$$
\phi: A \rightarrow{ }_{p^{r}} F(R)
$$

are naturally in one-to-one correspondence with the set of Hopf algebra maps (over R)

$$
R[[x]] /\left(\left[p^{r}\right](x)\right) \rightarrow R^{A} .
$$

Proof. Unraveling the definitions, a map $\phi: A \rightarrow p^{n} F(R)$ corresponds to the Hopfalgebra homomorphism

$$
R[[x]] /\left(\left[p^{m}\right](x)\right) \rightarrow R^{A}
$$

whose $a$-component is the $R$-algebra extension of the $E^{*}$-algebra homomorphism corresponding to $\phi(a)$.

Proposition 6.2. The following conditions on a homomorphism

$$
\phi: \Lambda_{r}^{*} \rightarrow{ }^{r} F(R)
$$

are equivalent:

i) For all $\alpha \neq 0 \in \Lambda_{r}^{*}, \phi(\alpha)$ is a unit 12

ii) The Hopf algebra homomorphism

$$
R[[x]] /\left(\left[p^{r}\right](x)\right) \rightarrow R^{\Lambda_{r}^{*}}
$$

is an isomorphism.

The following two conditions are also equivalent:

$\left.\mathrm{i}^{\prime}\right)$ For all $\alpha \neq 0 \in \Lambda_{r}^{*}$, the element $\phi(\alpha)$ is not a zero-divisor.

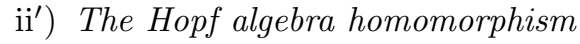

$$
R[[x]] /\left(\left[p^{r}\right](x)\right) \rightarrow R^{\Lambda_{r}^{*}}
$$

is a monomorphism.

Proof. With respect to the basis of powers of $x$ of the domain, and the obvious basis of the range, the matrix of the Hopf algebra homomorphism

$$
R[[x]] /\left(\left[p^{r}\right](x)\right) \rightarrow R^{\Lambda_{r}^{*}}
$$

is the Vandermonde matrix of the set $\phi\left(\Lambda_{r}^{*}\right)$. The result therefore follows from the first assertion of the next lemma.

In the following lemma, if $a$ and $b$ are elements of $R$, we will write $a \sim b$ if $a=\epsilon \cdot b$ where $\epsilon$ is a unit in $R$. We also let $\Delta$ denote the discriminant of the set $\phi\left(\Lambda_{r}^{*}\right)$, i.e.

$$
\Delta=\prod_{\alpha_{i} \neq \alpha_{j} \in \Lambda_{r}^{*}}\left(\phi\left(\alpha_{i}\right)-\phi\left(\alpha_{j}\right)\right) .
$$

\footnotetext{
${ }^{12}$ Thus $\phi$ is an isomorphism.
} 
Lemma 6.3. Let $\phi: \Lambda_{r}^{*} \rightarrow p^{r} F(R)$ be a homomorphism, as in the last lemma.

i)

$$
\Delta \sim \prod_{\alpha \neq 0 \in \Lambda_{r}^{*}} \phi(\alpha)^{\left|\Lambda_{r}^{*}\right|}
$$

ii) If the Hopf algebra map

$$
R[x]] /\left(\left[p^{r}\right](x)\right) \rightarrow R^{\Lambda_{r}^{*}}
$$

is a monomorphism, then

$$
\prod_{\alpha \neq 0 \in \Lambda_{r}^{*}} \phi(\alpha) \sim p^{r}
$$

so

$$
\Delta \sim p^{r p^{r n}}
$$

Proof. The formula

$$
x-y=(x-y) \cdot \epsilon(x, y), \quad \epsilon(x, y) \in E[[x, y]]^{\times},
$$

gives

$$
\begin{aligned}
\prod\left(\phi\left(\alpha_{i}\right)-\phi\left(\alpha_{j}\right)\right) & \sim \prod_{\alpha_{i}-\alpha_{j}=\alpha} \phi\left(\alpha_{i}-\alpha_{j}\right) \\
& =\prod_{\alpha \neq 0} \phi(\alpha) \\
& =\prod_{\alpha \neq 0} \phi(\alpha)^{\left|\Lambda_{r}^{*}\right|} .
\end{aligned}
$$

Now write

$$
\left[p^{r}\right](x)=g(x) \epsilon(x)
$$

with $g$ a monic polynomial of degree $p^{r n}$ and $\epsilon(x) \in E[x]^{\times}$. If the map (6.4) is a monomorphism, then, over $R$, there is a factorization

$$
g(x)=\prod_{\alpha \in \Lambda_{r}^{*}}(x-\phi(\alpha)) .
$$

Comparing coefficients of $x$ gives

$$
\prod_{\alpha \neq 0 \in \Lambda_{r}^{*}} \phi(\alpha)=\epsilon(0) \cdot p^{r} .
$$

6.2. The rings $L_{r}\left(E^{*}\right)$ and $L\left(E^{*}\right)$. If $R$ is an $E^{*}$-algebra, let $\mathcal{L}_{r}(R)$ be the set of all group homomorphisms

$$
\phi: \Lambda_{r}^{*} \rightarrow{ }_{p^{r}} F(R)
$$

satisfying either of the conditions [i) or [iil) of Proposition 6.2

Proposition 6.5. The functor $\mathcal{L}_{r}$ is representable by a ring $L_{r}\left(E^{*}\right)$ that is finite and faithfully flat over $p^{-1} E^{*}$. 
Proof. Let $\phi_{\text {univ }}: \Lambda_{r}^{*} \rightarrow p^{r} F\left(E^{*}\left(B \Lambda_{r}\right)\right)$ be the homomorphism corresponding to the identity map of $E^{*}\left(B \Lambda_{r}\right)$, and let $S \subset E^{*}\left(B \Lambda_{r}\right)$ be the multiplicatively closed subset generated by the $\phi(\alpha)$ with $\alpha \neq 0$. The functor $\mathcal{L}_{r}$ is represented by the ring

$$
L_{r}\left(E^{*}\right)=S^{-1} E^{*}\left(B \Lambda_{r}\right),
$$

which is clearly flat over $E^{*}$. Let $D_{r}\left(E^{*}\right)$ be the image of $E^{*}\left(B \Lambda_{r}\right)$ in $L_{r}\left(E^{*}\right)$. The ring $D_{r}\left(E^{*}\right)$ is finite over $E^{*}$, being a quotient of $E^{*}\left(B \Lambda_{r}\right)$. By Proposition 6.2 the Hopf algebra homomorphism

$$
D_{r}\left(E^{*}\right)\left[[x] /\left(\left[p^{r}\right](x)\right) \rightarrow D_{r}\left(E^{*}\right)^{\Lambda_{r}^{*}}\right.
$$

is a monomorphism. It follows from Lemma 6.3 that

$$
L_{r}\left(E^{*}\right)=p^{-1} D_{r}\left(E^{*}\right),
$$

so $L_{r}\left(E^{*}\right)$ is finite and flat over $p^{-1} E^{*}$. To check that $L_{r}\left(E^{*}\right)$ is faithfully flat, we need to find, for each homogeneous prime ideal $\mathfrak{p}$ of $p^{-1} E^{*}$, a homogeneous prime $\mathfrak{q} \subset L_{r}\left(E^{*}\right)$ extending $\mathfrak{p}$. Choose a homomorphism $f$, with kernel $\mathfrak{p}$, from $p^{-1} E^{*}$ to an algebraically closed, graded field $L$. By Theorem [5.6, the group $p^{r} F(L)$ is isomorphic to $\Lambda_{r}^{*}$. A choice of an isomorphism determines a map

$$
\hat{f}: L_{r}\left(E^{*}\right) \rightarrow L
$$

extending $f$. The prime ideal ker $\hat{f}$ is the desired $\mathfrak{q}$.

The group $\operatorname{Aut}\left(\Lambda_{r}\right)$ acts naturally on $L_{r}\left(E^{*}\right)$, since it acts on the functor $\mathcal{L}_{r}$.

Proposition 6.6. The ring of invariants is just $p^{-1} E^{*}$.

Proof. To prove this, it suffices to find a faithfully flat $p^{-1} E^{*}$-algebra $R$, and show that the ring of $\operatorname{Aut}\left(\Lambda_{r}\right)$ invariants in $R \otimes_{E^{*}} L_{r}\left(E^{*}\right)$ is $R$.

For any $p^{-1} E^{*}$-algebra $R$, the ring $R \otimes_{E^{*}} L_{r}\left(E^{*}\right)$ represents the functor that assigns to each $R$-algebra $S$ the set of Hopf algebra isomorphisms (over $S$ )

$$
S\left[[x] /\left(\left[p^{r}\right](x)\right) \longrightarrow S^{\Lambda_{r}^{*}} .\right.
$$

Now choose a faithfully flat $R$ with the property that there is a Hopf algebra isomorphism

$$
R[[x]] /\left(\left[p^{r}\right](x)\right) \approx R^{\Lambda_{r}^{*}}
$$

(for example, $R=L_{r}\left(E^{*}\right)$ ). Then $R \otimes_{E^{*}} L_{r}\left(E^{*}\right)$ will represent the functor that assigns to each $R$-algebra $S$ the set of Hopf algebra automorphisms (over $S$ )

$$
S^{\Lambda_{r}^{*}} \longrightarrow S^{\Lambda_{r}^{*}}
$$

By the last part of the next lemma, we conclude that $R \otimes_{E^{*}} L_{r}\left(E^{*}\right)$ and $R^{\operatorname{Aut}\left(\Lambda_{r}\right)}$ are isomorphic, as they represent the same functor. But it is obvious that the ring of Aut $\left(\Lambda_{r}\right)$-invariants in $R^{\operatorname{Aut}\left(\Lambda_{r}\right)}$ is just $R$.

Lemma 6.7. Let $R$ be a ring.

i) Let $A$ and $B$ be finite sets. The functor (on the category of $R$-algebras)

$$
S \mapsto\left\{S \text {-algebra homomorphisms : } S^{A} \rightarrow S^{B}\right\}
$$

is represented by $R^{\mathrm{Hom}_{\mathrm{Set}}(B, A)}$. 
ii) Let $A$ and $B$ be finite abelian groups. The functor

$$
S \mapsto\left\{\text { Hopf algebra homomorphisms (over } S \text { ) : } S^{A} \rightarrow S^{B}\right\}
$$

is represented by $R^{\mathrm{Hom}_{\mathrm{Ab}}(B, A)}$.

iii) Let $A$ be a finite abelian group. The functor

$$
S \mapsto\left\{\text { Hopf algebra automorphisms (over } S \text { ) : } S^{A} \rightarrow S^{A}\right\}
$$

is represented by $R^{\mathrm{Aut}_{\mathrm{Ab}}(A)}$.

Proof. Let's first construct a natural transformation. Associate to

$$
R^{\mathrm{Hom}_{\text {Set }}(B, A)} \rightarrow S
$$

the map

$$
R^{A} \stackrel{R^{\text {eval. }}}{\longrightarrow} R^{B \times \operatorname{Hom}(B, A)} \approx R^{\operatorname{Hom}(B, A)^{B}} \rightarrow S^{B} .
$$

The map $S^{A} \rightarrow S^{B}$ is then extended by linearity. Note that this transformation is natural in $A$ and $B$. It is an isomorphism if $A$ is the one element set. It is therefore an isomorphism in general since both functors carry disjoint unions in $A$ to cartesian products, and we have proved the first part of the lemma.

The other two parts now follow from part (i) by naturality in $A$ and $B$.

A homomorphism

$$
\phi: \Lambda_{r}^{*} \rightarrow{ }_{p^{r}} F(R)
$$

satisfying one of the conditions of Proposition 6.2 restricts to a homomorphism

$$
\phi: \Lambda_{r-1}^{*} \rightarrow{ }_{p^{r-1}} F(R)
$$

satisfying the same condition. It follows that there are natural maps of $E^{*}$-algebras

$$
L_{r-1}\left(E^{*}\right) \rightarrow L_{r}\left(E^{*}\right) .
$$

Furthermore, this map will be $\operatorname{Aut}\left(\Lambda_{r}\right)$ equivariant, where the action on the domain is via the projection $\operatorname{Aut}\left(\Lambda_{r}\right) \rightarrow \operatorname{Aut}\left(\Lambda_{r-1}\right)$.

We let $L\left(E^{*}\right)$ be the colimit $\operatorname{colim}_{r} L_{r}\left(E^{*}\right) . L\left(E^{*}\right)$ will be acted on by the group $\operatorname{Aut}(\Lambda)$, and comes equipped with a canonical isomorphism of groups

$$
\phi_{\text {univ }}: \Lambda^{*} \rightarrow{ }_{p^{\infty}} F\left(L\left(E^{*}\right)\right) .
$$

Corollary 6.8. $\quad$ i) $L\left(E^{*}\right)$ represents the functor that assigns to each $E^{*}$-algebra $R$, the set of isomorphisms of groups

$$
\phi: \Lambda^{*} \rightarrow p^{\infty} F(R)
$$

such that $\phi(\alpha)$ is a unit for all $\alpha \neq 0 \in \Lambda_{r}^{*}$.

ii) $L\left(E^{*}\right)$ is faithfully flat over $p^{-1} E^{*}$.

iii) The ring of $\operatorname{Aut}(\Lambda)$ invariants is just $p^{-1} E^{*}$. 
6.3. Defining the generalized characters. In this subsection, we elaborate on the character ring constructions given in the introduction.

Choose $r$ large enough so that all $p$-torsion in $G$ has order dividing $p^{r}$. Then $\operatorname{Hom}\left(\Lambda_{r}, G\right)=G_{n, p}$, so that

$$
\operatorname{Fix}_{n, p}(G, X)=\coprod_{\alpha \in \operatorname{Hom}\left(\Lambda_{r}, G\right)} X^{\operatorname{Im}(\alpha)},
$$

where $X$ is a finite $G-\mathrm{CW}$ complex, and $\operatorname{Fix}_{n, p}(G, X)$ is as in the introduction. This fixed point space is a space with commuting actions of $G$ and $\operatorname{Aut}\left(\Lambda_{r}\right)$.

A homomorphism

$$
\alpha: \Lambda_{r} \rightarrow G
$$

induces a map

$$
\alpha: B \Lambda_{r} \times X^{\operatorname{Im}(\alpha)} \rightarrow E G \times_{G} X
$$

and thus a map

$$
\alpha^{*}: E^{*}\left(E G \times{ }_{G} X\right) \rightarrow E^{*}\left(B \Lambda_{r} \times X^{\operatorname{Im}(\alpha)}\right) \rightarrow L_{r}\left(E^{*}\right) \otimes_{E^{*}} E^{*}\left(X^{\operatorname{Im}(\alpha)}\right) .
$$

Taking the product over $\alpha$ yields a map

$$
\chi_{n, p}^{G}: E^{*}\left(E G \times_{G} X\right) \rightarrow L_{r}\left(E^{*}\right) \otimes_{E^{*}} E^{*}\left(\operatorname{Fix}_{n, p}(G, X)\right) .
$$

The codomain of $\chi_{n, p}^{G}$ admits an action of $G \times \operatorname{Aut}\left(\Lambda_{r}\right): G$ acts via its action on $\operatorname{Fix}_{n, p}(G, X)$, and $\operatorname{Aut}\left(\Lambda_{r}\right)$ acts diagonally on each of the factors in this tensor product.

Lemma 6.9. This map lands in the $G \times \operatorname{Aut}\left(\Lambda_{r}\right)$ invariants.

Proof. This compatibility is a consequence of geometric facts which have nothing to do with the cohomology theory $E^{*}$.

The invariance under the $\operatorname{Aut}\left(\Lambda_{r}\right)$ action follows from the commutative diagrams, for all $\alpha \in \operatorname{Hom}\left(\Lambda_{r}, G\right)$ and $\phi \in \operatorname{Aut}\left(\Lambda_{r}\right)$,

$$
\begin{array}{ccc}
B \Lambda_{r} \times X^{\operatorname{Im}(\alpha \circ \phi)} \stackrel{\phi \times 1}{\longrightarrow} B \Lambda_{r} \times X^{\operatorname{Im}(\alpha)} \\
\alpha \circ \phi \downarrow & \alpha \downarrow \\
E G \times_{G} X=E G \times_{G} X .
\end{array}
$$

To see the invariance under the action of $G$, suppose that $f: H \rightarrow G$ is a map of finite groups, $X$ a $G$-space, and consider the commutative diagram

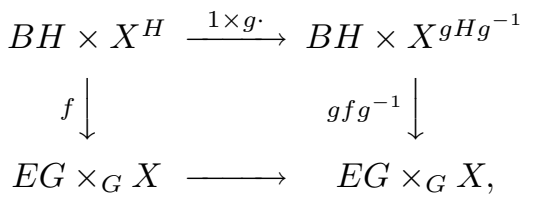

in which the bottom row comes from the map

$$
\begin{aligned}
(G, X) & \rightarrow(G, X) \\
(t, x) & \mapsto\left(g t g^{-1}, g x\right) .
\end{aligned}
$$

It is well known that the bottom map is homotopic to the identity Seg68:1. 
Because of the lemma, we conclude that $\chi_{n, p}^{G}$ induces

$$
\chi_{n, p}^{G}: E^{*}\left(E G \times_{G} X\right) \rightarrow C l_{n, p}\left(G, X ; L_{r}\left(E^{*}\right)\right)^{\operatorname{Aut}\left(\Lambda_{r}\right)},
$$

where

$$
C l_{n, p}\left(G, X ; L_{r}\left(E^{*}\right)\right)=L_{r}\left(E^{*}\right) \otimes_{E^{*}} E^{*}\left(\operatorname{Fix}_{n, p}(G, X)\right)^{G} .
$$

Also note that it is clear that these maps are compatible as $r$ varies, and, indeed, the codomain is independent of $r$ for all $r$ large enough.

6.4. Proof of Theorem $\mathbf{C}$. Consider the following properties of a functor $C=C^{*}$ from the category $\mathcal{C}$ of pairs $(G, X)$ to the category of graded abelian groups:

Mayer-Vietoris. On the category of pushout squares

$$
\begin{array}{ccc}
W & \rightarrow & X \\
\downarrow & & \downarrow \\
Y & \rightarrow & Z,
\end{array}
$$

in $\mathcal{C}$, there is a natural connecting homomorphism

$$
\delta: C^{n}(W) \rightarrow C^{n+1}(Z), \quad n \in \mathbb{Z},
$$

giving rise to a long exact Mayer-Vietoris sequence

$$
\cdots \rightarrow C^{*}(Z) \rightarrow C^{*}(Y) \oplus C^{*}(X) \rightarrow C^{*}(W) \stackrel{\delta}{\rightarrow} C^{*+1}(Z) \rightarrow \cdots .
$$

Induction. For any $H \subset G$ and $H$-space $Y$, the natural map

$$
(H, Y) \rightarrow\left(G, G \times{ }_{H} Y\right)
$$

induces an isomorphism

$$
C^{*}\left(G, G \times_{H} Y\right) \approx C^{*}(H, Y) .
$$

Descent. Let $F$ be the bundle of flags in an equivariant complex vector bundle over $X$. The sequence

$$
X \leftarrow F \leftleftarrows F \times{ }_{X} F
$$

gives rise to an equalizer diagram

$$
C^{*}(G, X) \rightarrow C^{*}(G, F) \rightrightarrows C^{*}\left(G, F \times_{X} F\right) .
$$

Lemma 6.10. Let $\tau: C \rightarrow D$ be a natural transformation between homotopy functors satisfying the above three properties. Suppose that $\tau$ also commutes with the connecting homomorphisms of the Mayer-Vietoris sequences. If $\tau(A, p t)$ is an isomorphism for all abelian groups $A$, then $\tau(G, X)$ is an isomorphism for all finite groups $G$, and all finite $G-C W$ complexes $X$.

Proof. First use descent to conclude that $\tau(G, X)$ is an isomorphism if $\tau(G, X \times F(V))$ is an isomorphism, where $F(V)$ is the manifold of complete flags in a faithful complex representation of $G$. We may therefore assume that the only subgroups of $G$ which fix a point of $X$ are abelian. Next run an induction on the dimension of $X$, applying the Mayer-Vietoris sequences to an equivariant cell decomposition, to show that $\tau(G, X)$ is an isomorphism if $\tau\left(G, G / H \times D^{n}\right)$ is an isomorphism for all $n \geq 0$ and all $H \subseteq G$ which fix a point of $X$. We have reduced to showing that $\tau\left(G, G / A \times D^{n}\right)$ is an isomorphism for all abelian $A \subseteq G$ and all $n \geq 0$. By homotopy invariance, this is the same as showing that $\tau(G, G / A)$ is an isomorphism, and by the induction property, this is equivalent to showing that $\tau(A, \mathrm{pt})$ is an isomorphism for all abelian $A$. This completes the proof. 
Proof of Theorem $[$. The proof will be complete once it is established that the functors

$$
L\left(E^{*}\right) \otimes_{E^{*}} E^{*}\left(E G \times_{G} X\right) \quad \text { and } \quad L\left(E^{*}\right) \otimes_{E^{*}} E^{*}\left(\operatorname{Fix}_{n, p}(G, X)\right)^{G}
$$

have the above three properties, the transformation $\chi_{n, p}^{G}$ commutes with the connecting homomorphisms of the Mayer-Vietoris sequences, and finally that $\chi_{n, p}^{A}$ is an isomorphism when $X$ is a point.

Mayer-Vietoris. The Mayer-Vietoris sequences come from the usual MayerVietoris sequences of the pushout squares

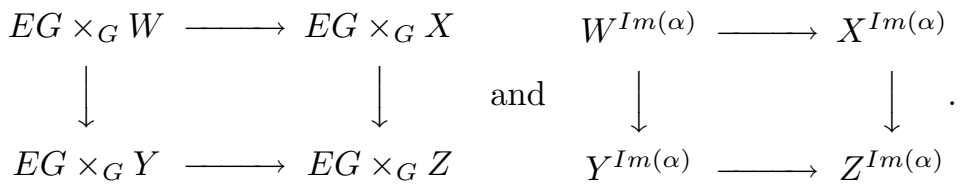

The connecting homomorphisms clearly commute with $\chi_{n, p}^{G}$. The sequences are exact because

(1) $L\left(E^{*}\right)$ is flat over $E^{*}$

(2) The order of $G$ is a unit in $L\left(E^{*}\right)$, so passage to $G$-invariants is exact.

Induction. Since the map

$$
(H, Y) \rightarrow\left(G, G \times_{H} Y\right)
$$

gives rise to a homotopy equivalence

$$
E H \times_{H} Y \simeq E G \times_{G} G \times_{H} Y,
$$

the functor $L\left(E^{*}\right) \otimes_{E^{*}} E^{*}\left(E G \times_{G} X\right)$ has the induction property.

The induction property for $L\left(E^{*}\right) \otimes_{E^{*}} E^{*}\left(\operatorname{Fix}_{n, p}(G, X)\right)^{G}$ follows from the observation that there is a natural homeomorphism of $G$-spaces

$$
G \times_{H} \operatorname{Fix}_{n, p}(H, Y) \simeq \operatorname{Fix}_{n, p}\left(G, G \times_{H} Y\right) .
$$

See [Kuh89] for more about the properties of such fixed point functors.

Descent. The descent property follows from Proposition 2.6, the flatness of $L\left(E^{*}\right)$, and the fact that passage to $G$-invariants is exact.

Finally, we need to verify that

$$
\chi_{n, p}^{A}: L\left(E^{*}\right) \otimes_{E^{*}} E^{*}(B A) \rightarrow L\left(E^{*}\right)^{\operatorname{Hom}(\Lambda, A)}
$$

is an isomorphism for every finite abelian group $A$.

Since both domain and range of the character map convert products of abelian groups into tensor products, it suffices to consider the case when $A$ is cyclic. In particular, it is convenient to let $A=\left(\mathbb{Z} / p^{r}\right)^{*}$. In this case, $\operatorname{Hom}(\Lambda, A)$ is canonically isomorphic to $\Lambda_{r}^{*}, E^{*}(B A)$ is canonically isomorphic to $\left.E^{*}[x]\right] /\left(\left[p^{r}\right](x)\right)$, and $\chi_{n, p}^{A}$ identifies with the canonical isomorphism

$$
L\left(E^{*}\right)[[x]] /\left(\left[p^{r}\right](x)\right) \simeq L\left(E^{*}\right)^{\Lambda_{r}^{*}} .
$$

This completes the proof of Theorem $\mathrm{C}$. 
Remark 6.11. Here is a second proof of the isomorphism

$$
\chi_{n, p}^{G}: L\left(E^{*}\right) \otimes_{E^{*}} E^{*}(B G) \simeq C l_{n, p}\left(G ; L\left(E^{*}\right)\right)
$$

that doesn't use the flatness of $L\left(E^{*}\right)$ : Theorem 3.3 applies to the functor

$$
h(X)=L\left(E^{*}\right) \otimes_{E^{*}} E^{*}\left(E G \times_{G} X\right),
$$

thus

$$
L\left(E^{*}\right) \otimes_{E^{*}} E^{*}(B G) \simeq \lim _{A \in \mathcal{A}(G)} L\left(E^{*}\right) \otimes_{E^{*}} E^{*}(B A) .
$$

But since it is clear that $G_{n, p}=\operatorname{colim}_{A \in \mathcal{A}(G)} A_{n, p}$, we have

$$
C l_{n, p}\left(G ; L\left(E^{*}\right)\right) \simeq \lim _{A \in \mathcal{A}(G)} C l_{n, p}\left(A ; L\left(E^{*}\right)\right) .
$$

One is reduced to checking that the character map is an isomorphism in the abelian group case, and one proceeds as before.

6.5. Induction. In this subsection we establish Theorem $\mathrm{D}$, the induction formula for characters.

We will use four properties of the transfer associated to a finite covering $W \rightarrow Z$ Ada78, Chapter 4]:

(1) the transfer associated to the identity map is the identity map;

(2) if $W_{1} \amalg W_{2} \rightarrow Z$ is a disjoint union of finite coverings, then the transfer map

$$
E^{*}\left(W_{1}\right) \oplus E^{*}\left(W_{2}\right) \rightarrow E^{*}(Z)
$$

is the sum of the transfer maps associated to the coverings $W_{1} \rightarrow Z$ and $W_{2} \rightarrow Z$;

(3) the transfer $E^{*}(Z) \rightarrow E^{*}(W)$ is a map of $E^{*}(Z)$-modules;

(4) if

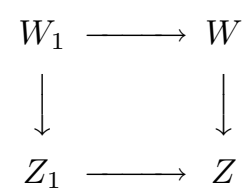

is a fiber square, then the diagram

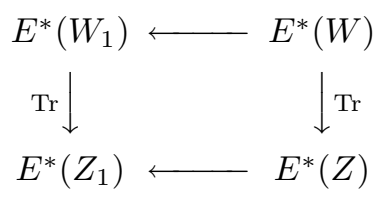

commutes.

Lemma 6.12 (Compare with Die72], Satz 4). If $A \subset \Lambda_{r}$ is a proper subgroup, the composite

$$
E^{*}(B A) \stackrel{\operatorname{Tr}}{\longrightarrow} E^{*}\left(B \Lambda_{r}\right) \rightarrow L_{r}\left(E^{*}\right)
$$

is zero.

Proof. Recall that $L_{r}\left(E^{*}\right)=S^{-1} E^{*}\left(B \Lambda_{r}\right)$, where $S$ is the image of the nonzero elements of $\Lambda_{r}^{*}$ under the canonical map

$$
\phi_{\text {univ }}: \Lambda_{r}^{*} \rightarrow E^{*}\left(B \Lambda_{r}\right) .
$$

Choose a nontrivial $\alpha$ in the kernel of $\Lambda_{r}^{*} \rightarrow A^{*}$, and let $x=\phi_{\text {univ }}(\alpha) \in E^{*}\left(B \Lambda_{r}\right)$. By construction, $x$ restricts to 0 in $E^{*}(B A)$, thus multiplication by $x$ annihilates the image of the transfer by property (3). But $x$ becomes a unit in $L_{r}\left(E^{*}\right)$. 
Corollary 6.13. Suppose that $Y$ is a trivial $\Lambda_{r}-$ space, and that $J$ is a finite $\Lambda_{r}-$ set with

$$
J^{\Lambda_{r}}=\emptyset
$$

Then the composite

$$
E^{*}\left(E \Lambda_{r} \times_{\Lambda_{r}}(J \times Y)\right) \stackrel{\operatorname{Tr}}{\longrightarrow} E^{*}\left(B \Lambda_{r} \times Y\right) \rightarrow L_{r}\left(E^{*}\right) \otimes_{E^{*}} E^{*}(Y)
$$

is zero.

Proof. By property (2) we reduce to the case

$$
J=\Lambda_{r} / A, \quad A \neq \Lambda_{r} .
$$

Properties (3) and (4) and Corollary 5.11 show in this case that (6.14) is just the tensor product (over $E^{*}$ ) of the identity map of $E^{*}(Y)$ with

$$
E^{*}(B A) \stackrel{\operatorname{Tr}}{\longrightarrow} E^{*}\left(B \Lambda_{r}\right) \rightarrow L_{r}\left(E^{*}\right)
$$

But this map is zero by the lemma.

Proof of Theorem $D$. Let $H$ be a subgroup of $G, X$ a $G$-space, and $x$ an element of $E^{*}\left(E H \times_{H} X\right)=E^{*}\left(E G \times_{G}\left(G \times_{H} X\right)\right)$. If $\alpha \in \operatorname{Hom}(\Lambda, G)$ factors through $\Lambda \rightarrow \Lambda_{r}$, we can calculate the $\chi_{n, p}^{G}(\alpha)\left(\operatorname{Tr}^{*}(x)\right) \in L\left(E^{*}\right) \otimes_{E^{*}} E^{*}\left(X^{I m(\alpha)}\right)$ by applying property (4) to the pullback diagram

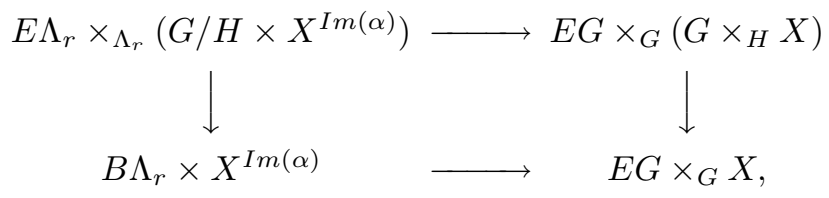

which is the composite of the pullback diagrams

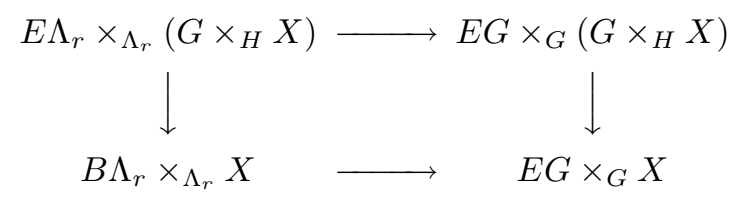

and

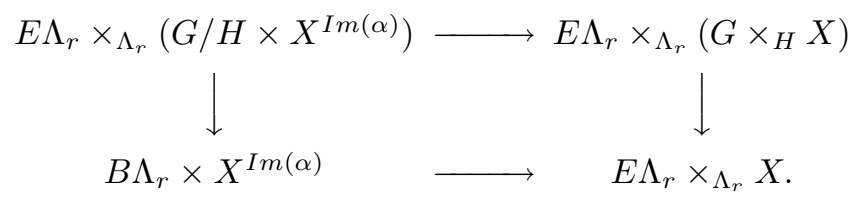

Let $J$ be the complement of $(G / H)^{\operatorname{Im}(\alpha)}$ in $G / H$, so that

$$
J^{\Lambda_{r}}=\emptyset \text {. }
$$

The space $E \Lambda_{r} \times_{\Lambda_{r}}\left(G / H \times X^{\operatorname{Im}(\alpha)}\right)$ decomposes into the disjoint union of

$$
\coprod_{g H \in(G / H)^{\operatorname{Im}(\alpha)}} B \Lambda_{r} \times\{g H\} \times X^{\operatorname{Im}(\alpha)} \text { and } E \Lambda_{r} \times{ }_{\Lambda_{r}} J \times X^{\operatorname{Im}(\alpha)} .
$$

The image of $x$ under the composite

$$
\begin{array}{rcc}
E^{*}\left(E G \times_{G}\left(G \times_{H} X\right)\right) & \rightarrow & E^{*}\left(B \Lambda_{r} \times\{g H\} \times X^{\operatorname{Im}(\alpha)}\right) \\
\stackrel{\operatorname{Tr}}{\rightarrow} & E^{*}\left(B \Lambda_{r} \times X^{\operatorname{Im}(\alpha)}\right) \\
& \rightarrow & L_{r}\left(E^{*}\right) \otimes_{E^{*}} E^{*}\left(X^{\operatorname{Im}(\alpha)}\right)
\end{array}
$$


is $\chi_{n, p}^{H}(g \cdot \alpha)(x)$. The composite

$$
E^{*}\left(E \Lambda_{r} \times \Lambda_{r}\left(J \times X^{f}\right)\right) \stackrel{\operatorname{Tr}}{\longrightarrow} E^{*}\left(B \Lambda_{r} \times X^{f}\right) \rightarrow L_{r}\left(E^{*}\right) \otimes_{E^{*}} E^{*}\left(X^{\operatorname{Im}(\alpha)}\right)
$$

is zero by Corollary 6.13. Theorem $D$ now follows from property (2) of the transfer.

Remark 6.15. The key idea in this proof is our use of property (3) in the proof of Lemma 6.12. This can be similarly used to get a quick derivation of a formula of tom Dieck Die72]. Let $C_{m}=\mathbb{Z} / m$. Then, for any $k, m$, and complex oriented theory $E^{*}$ with orientation $x$, one has

$$
\operatorname{Tr}(1)=[m k](x) /[m](x) \in E^{*}\left(B C_{m k}^{*}\right)
$$

where

$$
\operatorname{Tr}: E^{*}\left(B C_{m}^{*}\right) \rightarrow E^{*}\left(B C_{m k}^{*}\right)
$$

is the transfer associated to $C_{m}^{*} \subset C_{m k}^{*}$. The proof goes as follows. First, one can assume $E^{*}=M U^{*}$, so that $E^{*}\left(B C_{m k}\right)=E^{*}[[x] / /([m k](x))$. Arguing as in Lemma 6.12, one deduces that $\operatorname{Tr}(1)$ will satisfy: $\operatorname{Tr}(1)$ is annihilated by multiplication by $[m](x)$, and $\operatorname{Tr}(1) \equiv k \bmod x$. But the only element in $E^{*}[[x] /([m k](x))$ satisfying these two properties is $[m k](x) /[m](x)$.

Example 6.16. As an illustration of our induction formula, we compute the map

$$
A(G) \rightarrow \pi^{0}(B G) \rightarrow M U^{*}(B G) \rightarrow E^{*}(B G) \rightarrow C l_{n, p}\left(G ; L\left(E^{*}\right)\right),
$$

i.e. the Hurewitz map from the Burnside ring of $G$ to our ring of characters. Given a virtual finite $G$-set $S$, and $\alpha \in G_{n, p}$, the formula is

$$
\chi_{n, p}^{G}(\alpha)(S)=\left|S^{\operatorname{Im}(\alpha)}\right| .
$$

To verify this, it suffices to assume that $S=G / H$. In this case, the image of $S$ in $E^{*}(B G)$ will be $\operatorname{Tr}(1)$, and Theorem $\mathrm{D}$ shows that

$$
\chi_{n, p}^{G}(\alpha)(\operatorname{Tr}(1))=\left|(G / H)^{\operatorname{Im}(\alpha)}\right| .
$$

In particular, we deduce that

$$
\operatorname{Ker}\left\{A(G) \rightarrow M U^{*}(B G)\right\} \subseteq\left\{S|| S^{A} \mid=0 \text { for all abelian } A \subset G\right\} .
$$

But Theorem A implies that the other inclusion is true up to finite index.

\section{GoOd GROups}

In this section, we fix a prime $p$ and $n>0$, and study the question of whether $K(n)^{*}(B G)$ is concentrated in even degrees via the notion of good groups.

\subsection{Good groups and the wreath product theorem.}

Definition 7.1. (1) For a finite group $G$, an element

$$
x \in K(n)^{*}(B G)
$$

is good if it is a transferred Euler class of a complex subrepresentation of $G$, i.e. a class of the form $\operatorname{Tr}_{H}^{G}(e(\rho))$ where $\rho$ is a complex representation of a subgroup $H<G$, and $e(\rho) \in K(n)^{*}(B H)$ is its Euler class (i.e. its top Chern class).

(2) $G$ is good if $K(n)^{*}(B G)$ is spanned by good elements as a $K(n)^{*}-$ module. 
Note that, in general, $\operatorname{Tr}_{H}^{G}(e(\rho)) \neq e\left(\operatorname{Ind}_{H}^{G}(\rho)\right)$. For good $G, K(n)^{*}(B G)$ cannot be described only in terms of representations of $G$ itself. If $G$ is good, then $K(n)^{*}(B G)$ is of course concentrated in even dimensions.

Elementary properties of good elements and good groups are summarized in the next proposition.

Proposition 7.2. i) Every finite abelian group is good.

ii) If $x_{1} \in K(n)^{*}\left(B G_{1}\right)$ and $x_{2} \in K(n)^{*}\left(B G_{2}\right)$ are both good, then so is $x_{1} \times x_{2} \in$ $K(n)^{*}\left(B\left(G_{1} \times G_{2}\right)\right)$. Thus if $G_{1}$ and $G_{2}$ are good, then so is their product $G_{1} \times G_{2}$. iii) $G$ is good if its $p$-Sylow subgroup is good.

iv) If $f: H \rightarrow G$ is a homomorphism and $x \in K(n)^{*}(B G)$ is good, then $f^{*}(x)$ is a linear combination of good elements in $K(n)^{*}(B H)$.

v) If $x$ and $y$ are good elements of $K(n)^{*}(B G)$, then their cup product $x y$ is a sum of good elements.

Proof. For statement i), we note that if $G$ is abelian, then the results in subsection 5.4 make it clear that $K(n)^{*}(B G)$ is generated by Euler classes of representations of $G$ itself.

Statement ii) is a consequence of the behavior of the transfer and Euler classes with respect to products: if $x_{1}=\operatorname{Tr}_{H_{1}}^{G_{1}}\left(e\left(\rho_{1}\right)\right)$ and $x_{2}=\operatorname{Tr}_{H_{2}}^{G_{2}}\left(e\left(\rho_{2}\right)\right)$, then $x_{1} \times x_{2}=$ $\operatorname{Tr}_{H_{1} \times H_{2}}^{G_{1} \times G_{2}}\left(e\left(\rho_{1} \oplus \rho_{2}\right)\right)$.

Statement iii) follows from the fact that $\operatorname{Tr}: K(n)^{*}\left(B G_{(p)}\right) \rightarrow K(n)^{*}(B G)$ is onto.

To prove statement iv), suppose $x=\operatorname{Tr}(e(\rho))$ where $\rho$ is a representation of $K<G$. Then there is a pullback diagram of the form

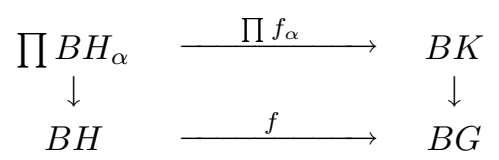

where each $H_{\alpha}$ is a subgroup of $H$. By naturality of the transfer,

$$
f^{*}(x)=\sum_{\alpha} \operatorname{Tr}\left(e\left(f_{\alpha}^{*}(\rho)\right)\right) .
$$

Finally, statement v) is a consequence of ii) and iv): $x \times y \in K(n)^{*}(B(G \times G))$ is good and $x y=\Delta^{*}(x \times y)$ where $\Delta: G \rightarrow G \times G$ is the diagonal map.

The next theorem is the main result of this section.

Theorem 7.3. If a finite group $G$ is good, then so is the wreath product $W=$ $\mathbb{Z} / p \nmid G$.

To prove Theorem [7.3, we study the extension

$$
1 \rightarrow G^{p} \rightarrow W \stackrel{\pi}{\longrightarrow} \mathbb{Z} / p \rightarrow 1
$$

and the associated spectral sequence $\left\{E_{r}^{*, *}(B W)\right\}$ with

$$
E_{2}^{*, *}(B W)=H^{*}\left(\mathbb{Z} / p ; K(n)^{*}\left(B G^{p}\right)\right) \Rightarrow K(n)^{*}(B W) .
$$

$\mathbb{Z} / p$ acts on $G^{p}$ by permuting the factors. Our first observation is that the induced action of $\mathbb{Z} / p$ on $K(n)^{*}\left(B G^{p}\right)$ makes $K(n)^{*}\left(B G^{p}\right)$ into a permutation module. This follows from the fact that the Künneth isomorphism

$$
K(n)^{*}(B G)^{\otimes p} \simeq K(n)^{*}\left(B G^{p}\right)
$$


is a $\mathbb{Z} / p$-module map. This is formal if $p$ is odd, as then $K(n)$ is a commutative ring spectrum. If $p=2, K(n)$ is no longer commutative; however a formula of Würgler Wur86, Prop.2.4] measures the deviation, and we conclude that (7.5) will still be a map of $\mathbb{Z} / p$-modules because of our hypothesis that $K(n)^{*}(B G)$ is concentrated in even degrees.

Thus, as a module over $\mathbb{Z} / p$, we have a decomposition

$$
K(n)^{*}\left(B G^{p}\right)=F \oplus T
$$

where $F$ is a free $\mathbb{Z} / p$-module and $T$ has trivial $\mathbb{Z} / p$-action. Moreover

$$
H^{i}(\mathbb{Z} / p ; F)= \begin{cases}F^{\mathbb{Z} / p} & \text { for } i=0, \\ 0 & \text { for } i>0,\end{cases}
$$

and

$$
H^{*}(\mathbb{Z} / p ; T)=H^{*}(B \mathbb{Z} / p) \otimes T .
$$

We recall that $E_{2}^{0, *}(B W)$ is isomorphic to $K(n)^{*}\left(B G^{p}\right)^{\mathbb{Z} / p}$ via the restriction $\operatorname{Res}_{G^{p}}^{W}: K(n)^{*}(B W) \rightarrow K(n)^{*}\left(B G^{p}\right)$. Via $\pi^{*}$, the spectral sequence $\left\{E_{r}^{*, *}(B W)\right\}$ is a module over the Atiyah-Hirzebruch spectral sequence $\left\{E_{r}^{*, *}(B \mathbb{Z} / p)\right\}$ that converges to $K(n)^{*}(B \mathbb{Z} / p)$.

Lemma 7.6. Every element in $E_{2}^{0, *}(B W)$ is a permanent cycle represented by a linear combination of good elements.

Assuming this lemma, it follows that for all $r \geq 2$, there are isomorphisms of differential graded $K(n)^{*}$-vector spaces

$$
\left(E_{r}^{*, *}(\mathbb{Z} / p) \otimes T\right) \oplus F^{\mathbb{Z} / p} \rightarrow E_{r}^{*, *}(B W) .
$$

Thus we conclude that $K(n)^{*}(B W)$ is spanned by products of the good elements of Lemma 7.6 and the image of $\pi^{*}: K(n)^{*}(B \mathbb{Z} / p) \rightarrow K(n)^{*}(B W)$. By parts i), iv), and v) of Proposition 7.2, $W$ is good, and we have proved Theorem 7.3

It remains to prove the lemma.

We begin by being more explicit about a decomposition of $\mathbb{Z} / p$-modules

$$
K(n)^{*}\left(B G^{p}\right)=F \oplus T .
$$

Choose a basis $\left\{x_{i}\right\}$ for $K(n)^{*}(B G)$. Let $F$ have basis $\left\{x_{i_{1}} \times \cdots \times x_{i_{p}}\right\}$ where the subscripts $i_{1}, \ldots, i_{p}$ are not all the same. Note that $F^{\mathbb{Z} / p}$ will then be spanned by elements of the form $N(y)$, where, if $y \in K(n)^{*}\left(B G^{p}\right), N(y)=\sum_{\sigma \in \mathbb{Z} / p} \sigma \cdot y$. Let $T$ have basis $\left\{P\left(x_{i}\right)\right\}$, where $P(x)=x \times \cdots \times x \in K(n)^{*}\left(B G^{p}\right)$ for $x \in K(n)^{*}(G)$. Lemma 7.6 then follows from the next two lemmas.

Lemma 7.7. If $y \in K(n)^{*}\left(B G^{p}\right)$ is good, there is a good element $z \in K(n)^{*}(B W)$ so that $\operatorname{Res}_{G^{p}}^{W}(z)=N(y)$.

Lemma 7.8. If $x \in K(n)^{*}(B G)$ is good, there is a good element $z \in K(n)^{*}(B W)$ so that $\operatorname{Res}_{G^{p}}^{W}(z)=P(x)$.

Proof of Lemma 7.7. As $G^{p}$ is normal in $W$, we have $\operatorname{Res}_{G^{p}}^{W}\left(\operatorname{Tr}_{G^{p}}^{W}(y)\right)=N(y)$. Thus one can let $z=\operatorname{Tr}_{G^{p}}^{W}(y)$.

Proof of Lemma 7.8. Suppose $x$ is the transferred Euler class $\operatorname{Tr}(e(\rho))$ for a complex representation $\rho$ of some subgroup $H<G$. The representation $\rho \oplus \cdots \oplus \rho$ of $H^{p}$ extends to a representation $\hat{\rho}$ of $W=\mathbb{Z} / p \curlywedge H$ and $e(\hat{\rho})$ restricts to $P(e(\rho))$. 
There is a commutative diagram

$$
\begin{array}{cccc}
K(n)^{*}\left(B H^{p}\right) & \stackrel{\text { Res }}{\longleftarrow} & K(n)^{*}(B(\mathbb{Z} / p \nmid H)) \\
\downarrow & & \begin{array}{c}
\downarrow \operatorname{Tr} \\
\operatorname{Tr}
\end{array} \\
K(n)^{*}\left(B G^{p}\right) & \stackrel{\text { Res }}{\longleftarrow} & K(n)^{*}(B W) .
\end{array}
$$

Hence we have

$$
\begin{aligned}
\operatorname{Res}(\operatorname{Tr}(e(\hat{\rho}))) & =\operatorname{Tr}(\operatorname{Res}(e(\hat{\rho}))) \\
& =\operatorname{Tr}(P(e(\rho))) \\
& =P(\operatorname{Tr}(e(\rho))) \\
& =P(x) .
\end{aligned}
$$

So we can take $z=\operatorname{Tr}(e(\hat{\rho}))$.

7.2. The nonabelian groups of order $p^{3}$. Next we recall the results of TezukaYagita $[\mathrm{TY}]$ and explain how their work shows that $G$ is good for each nonabelian group of order $p^{3}$. For each prime there are two such groups, and in each case there is an extension

$$
1 \longrightarrow \mathbb{Z} / p \longrightarrow G \longrightarrow(\mathbb{Z} / p)^{2} \longrightarrow 1 \text {. }
$$

Let $c \in G$ be a generator of the subgroup of order $p$, which is the center $C$ of $G$, and let $a, b \in G$ be elements which map to generators of the quotient group, which is the abelianization of $G$. In each case we have relations $c=[a, b]$ and $c^{p}=1$. For $p$ odd we can take $b^{p}=1$ and $a^{p}=1$ or $c$; these two groups are denoted by $E$ and $M$, respectively. For $p=2$ we can take $a^{2}=c$ and $b^{2}=1$ or $c$; these are the dihedral group $D_{8}$ and the quaternion group $Q_{8}$, respectively.

Consider the abelian subgroup $A<G$ generated by $b$ and $c$. In the quaternion case it is cyclic of order four generated by $b$, and in the other three cases it is elementary abelian of rank 2. Define a one-dimensional representation $\phi$ of $A$ by $\phi(b)=i$ in the quaternion case and $\phi(c)=e^{2 \pi i / p}, \phi(b)=1$ in the other cases. Let $\rho$ be the representation of $G$ induced by $\phi$ and let $c_{1}, \ldots, c_{p} \in K(n)^{*}(B G)$ be its Chern classes.

Let $\lambda_{1}$ and $\lambda_{2}$ be two multiplicative generators of the representation ring of the quotient and let $y_{1}, y_{2} \in K(n)^{*}(B G)$ denote the images of their Euler classes.

Then we have

Theorem 7.9 ([TY] $)$. Let $G$ be a nonabelian group of order $p^{3}$. Then $K(n)^{*}(B G)$ is multiplicatively generated by the classes

$$
y_{1}, y_{2}, c_{1}, \ldots, c_{p}
$$

defined above. A similar statement holds for $B P^{*}(B G)$ and there is an isomorphism

$$
K(n)^{*}(B G) \simeq K(n)^{*} \otimes_{B P^{*}} B P^{*}(B G) .
$$

Moreover, the generators $c_{1}, \ldots, c_{p}$ can be replaced by any other elements $x_{1}, \ldots, x_{p}$ such that $x_{i}$ and $c_{i}$ have the same restriction in $B P^{*}(B C)$.

We assume this calculation and note that $y_{1}, y_{2}$, and $c_{p}$ are Euler classes of representations of $G$. By Proposition 7.2 if we can find elements $x_{1}, \ldots, x_{p-1}$ as in the theorem that are also transferred Euler classes, it will follow that $G$ is good.

Let $v \in B P^{*}(B A)$ be the Euler class of $\phi$ and $u \in B P^{*}(B C)$ its restriction. The total Chern class of $\rho$ restricts to $(1+u)^{p}$, i.e. $c_{i}$ restricts to a unit multiple of $p u^{i}$ for $1 \leq i \leq p-1$. Similarly, by the double coset formula we see that 
$x_{i}=\operatorname{Tr}\left(v^{i}\right) \in B P^{*}(B G)$ restricts to $p u^{i} \in B P^{*}(B C)$. Since $v^{i}$ is an Euler class, $y_{1}, y_{2}, x_{1}, \ldots, x_{p-1}, c_{p}$ are good elements that generate $B P^{*}(B G)$. We have proved

Proposition 7.10. Every group of order $p^{3}$ is good in the sense of 7.1 .

\section{ACKNOWLEDGEMENTS}

Many people deserve thanks for their interest in this project. In particular, we thank the late J. F. Adams for supplying the second author with lemmas in stable rational equivariant homotopy used in section 3 and Andreas Dress for conversations on similar subjects, and Andrew Baker, Peter Landweber, and the referee of an earlier draft of this paper for steering us toward accurate hypotheses in Theorem C. The influence of Jack Morava on our work is also clear; he was certainly on the lookout for character theoretic interpretations of $E^{*}(B G)$ as early as any of us.

\section{REFERENCES}

[Ada74] J. F. Adams. Stable Homotopy and Generalised Homology. University of Chicago Press, Chicago, 1974. MR 53:6534

[Ada78] J. F. Adams. Infinite Loop Spaces. Number 90 in Annals of Mathematics Studies. Princeton University Press, Princeton, 1978. MR 80d:55001

[Ada82] J. F. Adams. Prerequisites (on equivariant stable homotopy) for Carlsson's Lecture. Springer Lecture Notes in Math., 1051:483-532, 1982. MR 86f:57037

[Ara82] S. Araki. Equivariant stable homotopy theory and idempotents of Burnside rings. Publ. RIMS, pages 1193-1212, 1982. MR 84g:57035

[Atiyah] M. F. Atiyah. Characters and cohomology of finite groups. Inst. Hautes Études Sci. Publ. Math., 9:23-64, 1961. MR 26:6228

[Ati67] M. F. Atiyah. K-theory. Benjamin Press, New York, 1967. MR 36:7130

[AS89] M. F. Atiyah and G. Segal. On equivariant Euler characteristics. J. Geom. Phys., 6:671677, 1989. MR 92c:19005

[BW89] A. Baker and U. Würgler. Liftings of formal groups and the Artinian completion of $v_{n}^{-1}$ BP. Math. Proc. Camb. Phil. Soc., 106:511-530, 1989. MR 90i:55008

[Bak98] A. Baker. Hecke algebras acting on elliptic cohomology. In Homotopy via algebraic geometry and group representations (Evanston, IL, 1997), A.M.S. Cont. Math. Series 220:17-26, 1998. MR 99h:55004

[BT] R. Bott and C. Taubes. On the rigidity theorems of Witten. J.A.M.S., 2:137-186, 1989. MR 89k:58270

[Die72] T. tom Dieck. Kobordismentheorie klassifizierender Räume und Transformationsgruppen, Math. Zeit., 126:31-39, 1972. MR 45:7744

[Die79] T. tom Dieck. Transformation Groups and Representation Theory, volume 766 of Lecture Notes in Mathematics. Springer-Verlag, New York, 1979. MR 82c:57025]

[TtD:87] T. tom Dieck. Transformation Groups, volume 8 of de Gruyer Studies in Mathematics. de Gruyer, New York, 1987. MR 89c:57048

[Haz78] M. Hazewinkel. Formal Groups and Applications. Academic Press, New York, 1978. MR 82a: 14020

[Hop89] M. J. Hopkins. Characters and elliptic cohomology. In S. M. Salamon, B. Steer, and W. A. Sutherland, editors, Advances in Homotopy, volume 139 of London Mathematical Society Lecture Note Series, pages 87-104, Cambridge, 1989. Cambridge University Press. MR 91c:55007

[HKR92] M. J. Hopkins, N. J. Kuhn, and D. C. Ravenel. Morava $K$-theories of classifying spaces and generalized characters for finite groups. Algebraic Topology (San Feliu de Guixols, 1990), Springer Lecture Notes in Math., 1509:186-209, 1992. MR 93k:55008

[Hun90] J. R. Hunton. The Morava $K$-theories of wreath products. Math. Proc. Cambridge Phil. Soc., 107:309-318, 1990. MR 91a:55004 
[Ill74] S. Illman. Whitehead torsion and group actions. Ann. Acad. Sc. Fennicae, 588, 1974. MR 51:1407

[Kri97] I. Kriz. Morava $K$-theory of classifying spaces: some calculations. Topology, 36:12471273, 1997. MR 99a:55016

[KL98] I. Kriz and K. P. Lee. Odd degree elements in the Morava $K(n)$ cohomology of finite groups. Preprint, 1998. To appear in Topology and its applications.

[Kuhn1] N. J. Kuhn. Morava K-theories of some classifying spaces. Transactions of the American Mathematical Society, 304:193-205, 1987. MR 89d:55013

[Kuh89] N. J. Kuhn. Character rings in algebraic topology. In S. M. Salamon, B. Steer, and W. A. Sutherland, editors, Advances in Homotopy, volume 139 of London Mathematical Society Lecture Note Series, pages 111-126, Cambridge, 1989. Cambridge University Press. MR 91g:55023

[Lai79] E. Laitinen. On the Burnside ring and stable cohomotopy of a finite group. Math. Scand., 44:37-72, 1979. MR 80k:55030

[Lan71] P. S. Landweber. Complex cobordism of classifying spaces. Proceedings of the American Mathematical Society, 27:175-179, 1971. MR 42:3782

[LRS] P. S. Landweber, D. C. Ravenel, and R. E. Stong. Periodic cohomology theories defined by elliptic curves. In The Cech centennial (Boston, MA, 1993), A.M.S. Cont. Math. Series 181:317-337, 1995. MR 96i:55009

[Lan78] S. Lang. Cyclotomic Fields. Graduate Texts in Mathematics. Springer-Verlag, New York, 1978. MR 58:5578

[LMS86] L. G. Lewis, J. P. May, and M. Steinberger. Equivariant Stable Homotopy Theory, volume 1213 of Lecture Notes in Mathematics. Springer-Verlag, New York, 1986. MR 88e:55002

[LT65] J. Lubin and J. Tate. Formal complex multiplication in local fields. Annals of Mathematics, 81:380-387, 1965. MR 30:3094

[Mor78] J. Morava. Completions of complex cobordism. In M.G.Barrett and M.E.Mahowald, editors, Geometric Applications of Homotopy Theory II, Springer Lect. Notes Math. 658:349-361, 1978. MR 80i:57026

[Mor88] J. Morava. Some Weil representations motivated by algebraic topology. In P.S. Landweber, editor, Elliptic Curves and Modular Forms in Algebraic Topology, Springer Lect. Notes Math. 1326:94-106, 1988. MR 91a:57021

[Qui71] D. G. Quillen. The spectrum of an equivariant cohomology ring, I and II. Annals of Mathematics, 94:549-602, 1971. MR 45:7743

[Rav82] D. C. Ravenel. Morava K-theories and finite groups. In S. Gitler, editor, Symposium on Algebraic Topology in Honor of José Adem, Contemporary Mathematics, pages 289292, Providence, Rhode Island, 1982. American Mathematical Society. MR 83m:55009

[Rav86] D. C. Ravenel. Complex Cobordism and Stable Homotopy Groups of Spheres. Academic Press, New York, 1986. MR 87j:55003

[RW80] D. C. Ravenel and W. S. Wilson. The Morava $K$-theories of Eilenberg-Mac Lane spaces and the Conner-Floyd conjecture. American Journal of Mathematics, 102:691-748, 1980. MR 81i:55005

[Seg68:1] G. Segal. Classifying spaces and spectral sequences. Inst. Hautes Études Sci. Publ. Math., 34:105-112, 1968. MR 38:718

[Seg68:2] G. Segal. Equivariant K-theory. Inst. Hautes Études Sci. Publ. Math., 34:129-151, 1968. MR 38:2769

[Seg71] G. Segal. Equivariant stable homotopy theory. Actes Congr. Internat. Math., 2:59-63, 1970. MR 54:11319

[Serre] J.-P. Serre. Représentations Linéaires des Groupes Finis. Hermann, Paris, 1967. MR 38:1190

[Stre81] C. Stretch. Stable cohomotopy and cobordism of abelian groups. Math. Proc. Cambridge Phil. Soc., 90:273-278, 1981. MR 82h:55015

[Stri98] N. P. Strickland. Morava E-theory of symmetric groups. Topology, 37:757-779, 1998. MR 99e:55008; correction MR 2000a:55010

[Tan95] M. Tanabe. On Morava K-theories of Chevalley groups. Amer. J. Math., 117:263-278, 1995. MR 95m:55010

[Tau] C. Taubes. $S^{1}$-actions and elliptic genera. Comm. Math. Phys., 122:455-526, 1989. MR 90f:58167 
[TY] M. Tezuka and N. Yagita. Cohomology of finite groups and Brown-Peterson cohomology. In Algebraic Topology (Arcata, CA, 1986), Springer L.N.Math. 1370:396-408, 1989. MR 90i:55011

[Wur86] U. Würgler. Commutative ring spectra of characteristic 2. Comm. Math. Helv., 61:3345, 1986. MR 87i:55008

Department of Mathematics, Massachusetts Institute of Technology, Cambridge, MASSACHUSETTS 02139

E-mail address: mjh@math.mit.edu

Department of Mathematics, University of Virginia, Charlottesville, Virginia 22903

E-mail address: njk4x@virginia.edu

Department of Mathematics, University of Rochester, Rochester, New York 14627

E-mail address: drav@math.rochester.edu 